\title{
A convergent approach for the synthesis of new pyrazolyl bipyridinyl substituted coumarin derivatives as antimicrobials
}

\author{
Varun G. Bhila, Yogita L. Chovatiya, Chirag V. Patel, Rakesh R. Giri, \\ Dinkar I. Brahmbhatt* \\ Department of Chemistry, Sardar Patel University, Vallabh Vidyanagar - 388120, Gujarat, India \\ *Tel.: +912692 226856*219 \\ *E-mail address: drdib317@gmail.com
}

\begin{abstract}
Some new 3-[3-(1-phenyl-3-aryl-1H-pyrazol-4-yl)acryloyl]coumarins 3a-f were synthesized (coumarin chalcones) by the condensation of various 3 -acetyl coumarins 1 and appropriate 1-phenyl3-aryl-1H-pyrazole-4-carbaldehyde 2. These coumarin chalcones 3a-f were then employed for the synthesis of pyrazolyl bipyridinyl substituted coumarins 7a-f, 8a-f, and 9a-f under Krohnke's reaction condition. The characterization of all the synthesized compounds was carried out by elemental analysis, IR, ${ }^{1} \mathrm{H}-\mathrm{NMR},{ }^{13} \mathrm{C}-\mathrm{NMR}$, DEPT-135 and mass spectral analysis. In addition to that, in vitro antimicrobial competency of the title compounds was assessed against selected pathogens. Compounds $3 \mathbf{b}, \mathbf{3 e}, \mathbf{7 b}, \mathbf{8 b}, \mathbf{8 c}$ and $\mathbf{9 b}$ exhibited excellent antimicrobial activity and said to be the most proficient members of the series.
\end{abstract}

Keywords: Coumarin; Bipyridine; Pyrazole; Kröhnke’s reaction; Antimicrobial activity

\section{INTRODUCTION}

During past few decades, there has been an alarming increase in bacterial resistance to antibiotic drugs. The evolution and spread of these multidrug resistant bacteria have become a major threat to global health care [1].

Consequently, the discovery and design of new efficient antimicrobial agents is of crucial need to counteract the resistant pathogens. Therefore numerous research groups have directed their efforts towards designing the new anti-bacterial drugs with new targets, distinctive modes of action, low toxicity and low probability of inducing resistance to multi resistant bacteria [2-4].

Coumarin is extensively investigated as class of naturally occurring compounds and its derivatives have tremendous contribution in therapeutic field. Many coumarin derivatives used as effective drug in marketplace such as Warfarin, Novbiocin, Dicoumarol, Imperatorin, Calophyllolide and Neo-tarnshinlactone.

Such striking information from literature survey directed us to detail study of various heterocyclic substituted coumarin derivatives.

Among the coumarin derivatives, pyridyl substituted coumarins have been gained considerable interest among medicinal chemists owing to their therapeutic importance as CNS 
depressant [5], antifungal [6], moth proofing activity [7], fish toxicity [8], MAO inhibitor [9], antibacterial agents [10] and antitubercular [11].

During our literature survey we came across some bipyridines derivatives which have been reported to possess wide applications in the field of bioinorganic chemistry [12], supramolecular chemistry [13] and polymeric material [14]. Bipyridine derivatives exhibit wide range of physiological activities such as anticancer [15], cardiotonic [16], DNA binding properties [17] and antibacterial properties [18]. In addition to that, we came across some pyrazolyl substituted pyridines which have been reported to possess insecticidal [19], antiviral [20] and cardiotinic activities [21].

More efficacious antibacterial compounds can be designed by joining two or more biologically active heterocyclic systems together in a single molecular framework [22]. Such structural hybridizations show synergistic influence on the anticipated activity, hoping to discover new entities that would have astonishing antimicrobial activity.

In view of this background and medicinal significance of pyridyl substituted coumarins, bipyridines and pyrazolyl substituted pyridines encouraged us to hybrid these three bioactive moieties in single scaffold.

Hence, in continuation our efforts to synthesize such biologically active pyridyl substituted coumarin derivatives [10,11], we herein, report the synthesis and antimicrobial activity of pyrazolyl bipyridinyl coumarin derivatives7a-f, 8a-f, and 9a-f.

\section{RESULTS AND DISCUSSION}

\section{1. Chemistry}

The synthetic strategies adopted for the preparation of key precursor 3-[3-(1-phenyl-3aryl-1 $H$-pyrazol-4-yl)acryloyl]coumarins 3a-f and title compounds 7a-f, 8a-f and 9a-f were depicted in Scheme 1.

The title compounds 7a-f, 8a-f and 9a-f were synthesized by reacting appropriate 3-[3(1-phenyl-3-aryl-1H-pyrazol-4-yl)acryloyl]coumarins 3a-f with pyridoyl methyl pyridinium salts 4, 5 and $\mathbf{6}$ under Krohnke's reaction condition [23]. The required precursors 3a-f were prepared by the reaction of various 3 -acetyl coumarins $\mathbf{1}$ and pyrazole aldehydes $\mathbf{2}$ in presence of catalytical amount of piperidine in ethanol.

The plausible mechanism for the synthesis of target compounds 7a-f, 8a-f and 9a-f is demonstrated in Scheme 2.

The reaction proceeded via Michael addition by the nucleophilic attack of active methylene group of pyridoyl methyl pyridinium salts $(4,5$ and 6$)$ on the $\alpha, \beta$-unsaturated carbonyl functionality of 3-[3-(1-phenyl-3-aryl- $1 H$-pyrazol-4-yl)acryloyl]- coumarins 3a-f to form in situ 1,5-dicarbonyl intermediate.

The corresponding intermediate underwent cyclization in presence of ammonium acetate which upon subsequent loss of two water molecules afforded the target compounds in good yields.

All the compounds were characterized by IR, ${ }^{1} \mathrm{H}$ NMR, ${ }^{13} \mathrm{C}$ NMR, DEPT-90 spectral and elemental analysis. A mass spectrum was also recorded for representative compound $\mathbf{7 a}$. 

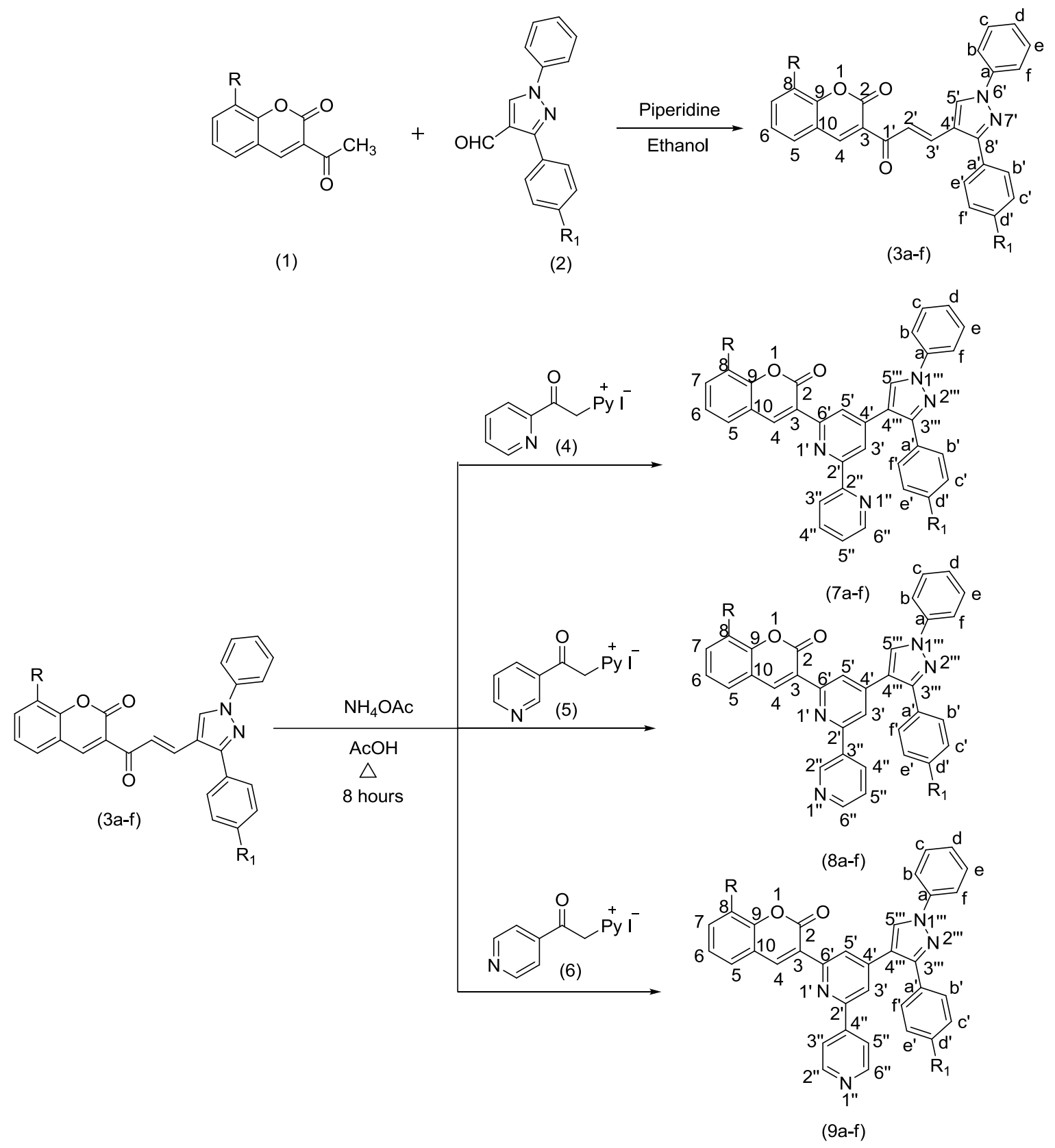

7a, $8 \mathrm{a}, 9 \mathrm{a}: \mathrm{R}=\mathrm{H}, \mathrm{R}_{1}=\mathrm{H}$

$7 \mathrm{~b}, 8 \mathrm{~b}, 9 \mathrm{~b}: \mathrm{R}=\mathrm{H}, \mathrm{R}_{1}=\mathrm{CH}_{3}$

7c, 8c, 9c: $\mathrm{R}=\mathrm{H}, \mathrm{R}_{1}=\mathrm{OCH}_{3}$ 7d, 8d, 9d : R= $\mathrm{OCH}_{3}, \mathrm{R}_{1}=\mathrm{H}$

7e, 8e, 9e: $\mathrm{R}=\mathrm{OCH}_{3}, \mathrm{R}_{1}=\mathrm{CH}_{3}$

7f, 8f, 9f: $\mathrm{R}=\mathrm{OCH}_{3}, \mathrm{R}_{1}=\mathrm{OCH}_{3}$

Scheme 1. Synthetic strategies adopted for the preparation of key precursors 3a-f and title compounds 7a-f, 8a-f and 9a-f. 

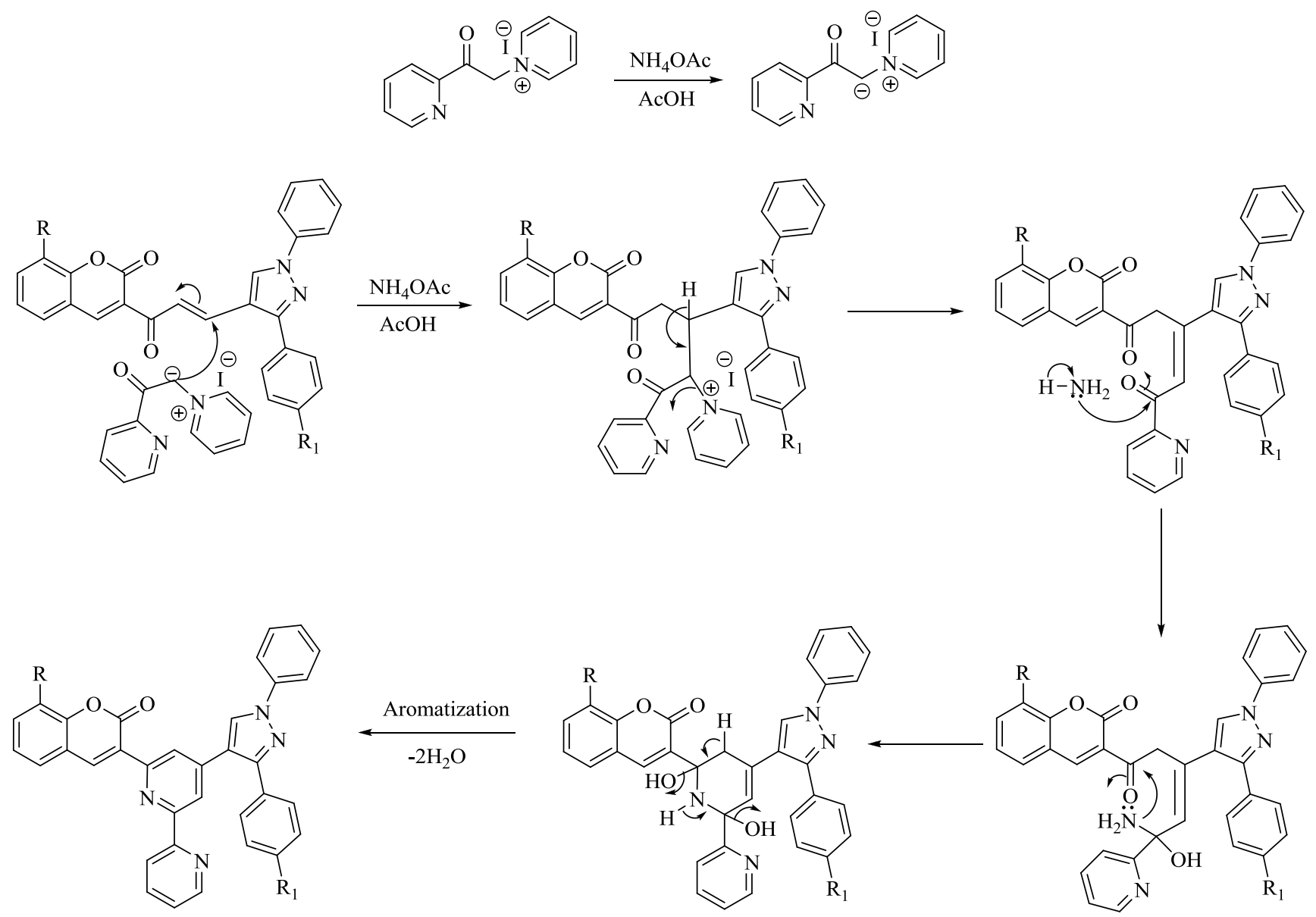

Scheme 2. Plausible mechanism for the synthesis of target compounds 7a-f, 8a-f and 9a-f.

\section{2. Biological result}

The MICs of synthesized compounds were determined out by broth microdilution method according to National Committee for Clinical Laboratory Standards (NCCLS, 2002) [24]. Upon reviewing antimicrobial data (Table 1) it has been observed that compounds $\mathbf{7 b}$ and 9b $(\mathrm{MIC}=50 \mu \mathrm{g} / \mathrm{mL})$ showed excellent activity against gram positive bacteria Staphylococcus aureus as compared to ampicillin ( $\mathrm{MIC}=250 \mu \mathrm{g} / \mathrm{mL})$. Similarly compounds 3e $(\mathrm{MIC}=50 \mu \mathrm{g} / \mathrm{mL})$ and $\mathbf{8 c}(\mathrm{MIC}=62.5 \mu \mathrm{g} / \mathrm{mL})$ showed excellent activity against gram positive bacteria Bacillus subtilis as compared to ampicillin ( $\mathrm{MIC}=250 \mu \mathrm{g} / \mathrm{mL}$ ). Compounds 3b, 8b $(\mathrm{MIC}=50 \mu \mathrm{g} / \mathrm{mL})$ and $7 \mathbf{b}(\mathrm{MIC}=62.5 \mu \mathrm{g} / \mathrm{mL})$ showed excellent activity against gram negative bacteria Escherichia coli and Salmonella typhi respectively as compared to ampicillin ( $\mathrm{MIC}=250 \mu \mathrm{g} / \mathrm{mL})$.

Compounds 3d, 7e, 8b, 9e (MIC $=100 \mu \mathrm{g} / \mathrm{mL})$ and 3a, 3e, 7a, 7d, 8a, 8e, 9a, 9c (MIC $=200 \mu \mathrm{g} / \mathrm{mL}$ ) were found to be more potent compared to ampicillin ( $\mathrm{MIC}=250 \mu \mathrm{g} / \mathrm{mL}$ ) against Staphylococcus aureus while 3b, 3f, 7c, 8c, 8d, 9d, 9f were found to be equipotent compared to ampicillin ( $\mathrm{MIC}=250 \mu \mathrm{g} / \mathrm{mL}$ ) against Staphylococcus aureus. Compounds $\mathbf{7 b}$, 9b $(\mathrm{MIC}=100 \mu \mathrm{g} / \mathrm{mL})$ and 3b, 3d, 7a, 7c, 8b, 8d, 9c $(\mathrm{MIC}=200 \mu \mathrm{g} / \mathrm{mL})$ were found to be more active while compounds 3a, 7f, 8f, 9e (MIC $=250 \mu \mathrm{g} / \mathrm{mL})$ were found to be equally active as compared to ampicillin (MIC $=250 \mu \mathrm{g} / \mathrm{mL}$ ) against Bacillus subtilis. 
Table 1. In vitro antimicrobial activity of various synthesized coumarin derivatives 3a-f, 7a-f, 8a-f and 9a-f (MICs, $\mu \mathrm{g} / \mathrm{mL})$.

\begin{tabular}{|c|c|c|c|c|c|c|c|c|}
\hline \multirow{2}{*}{\multicolumn{3}{|c|}{ Compounds }} & \multicolumn{2}{|c|}{ Gram positive bacteria } & \multicolumn{2}{|c|}{ Gram negative bacteria } & \multicolumn{2}{|c|}{ Fungi } \\
\hline & & & Sa. & Bs. & Ec. & St. & Ca. & An. \\
\hline & \multirow{2}{*}{$\mathbf{R}$} & \multirow{2}{*}{$\mathbf{R}_{1}$} & MTCC & MTCC & MTCC & MTCC & MTCC & MTCC \\
\hline & & & 96 & 441 & 443 & 98 & 227 & 282 \\
\hline $3 \mathbf{a}$ & $\mathrm{H}$ & $\mathrm{H}$ & 200 & 250 & 200 & 200 & 500 & $>1000$ \\
\hline $\mathbf{3 b}$ & $\mathrm{H}$ & $\mathrm{CH}_{3}$ & 250 & 200 & 50 & 200 & 100 & 500 \\
\hline $3 c$ & $\mathrm{H}$ & $\mathrm{OCH}_{3}$ & 500 & 500 & 250 & 250 & $>1000$ & 500 \\
\hline 3d & $\mathrm{OCH}_{3}$ & $\mathrm{H}$ & 100 & 200 & 250 & 200 & 250 & 250 \\
\hline $3 e$ & $\mathrm{OCH}_{3}$ & $\mathrm{CH}_{3}$ & 200 & 50 & 100 & 200 & 500 & 250 \\
\hline $3 f$ & $\mathrm{OCH}_{3}$ & $\mathrm{OCH}_{3}$ & 250 & 500 & 500 & 500 & 1000 & 1000 \\
\hline $7 \mathbf{a}$ & $\mathrm{H}$ & $\mathrm{H}$ & 200 & 200 & 250 & 200 & 500 & 500 \\
\hline $7 \mathbf{b}$ & $\mathrm{H}$ & $\mathrm{CH}_{3}$ & 50 & 100 & 100 & 62.5 & 1000 & 100 \\
\hline $7 \mathrm{c}$ & $\mathrm{H}$ & $\mathrm{OCH}_{3}$ & 250 & 200 & 200 & 250 & 250 & 500 \\
\hline $7 d$ & $\mathrm{OCH}_{3}$ & $\mathrm{H}$ & 200 & 500 & 250 & 500 & 500 & 500 \\
\hline $7 e$ & $\mathrm{OCH}_{3}$ & $\mathrm{CH}_{3}$ & 100 & 500 & 250 & 100 & 200 & 250 \\
\hline $7 f$ & $\mathrm{OCH}_{3}$ & $\mathrm{OCH}_{3}$ & 500 & 250 & 500 & 250 & $>1000$ & 500 \\
\hline $8 \mathbf{a}$ & $\mathrm{H}$ & $\mathrm{H}$ & 200 & 500 & 200 & 250 & 200 & 1000 \\
\hline $8 \mathbf{b b}$ & $\mathrm{H}$ & $\mathrm{CH}_{3}$ & 100 & 200 & 100 & 50 & 250 & 500 \\
\hline $8 \mathrm{c}$ & $\mathrm{H}$ & $\mathrm{OCH}_{3}$ & 250 & 62.5 & 200 & 200 & 200 & 250 \\
\hline $8 d$ & $\mathrm{OCH}_{3}$ & $\mathrm{H}$ & 250 & 200 & 100 & 250 & 500 & $>1000$ \\
\hline $8 \mathrm{e}$ & $\mathrm{OCH}_{3}$ & $\mathrm{CH}_{3}$ & 200 & 500 & 500 & 250 & 500 & $>1000$ \\
\hline $8 f$ & $\mathrm{OCH}_{3}$ & $\mathrm{OCH}_{3}$ & 500 & 250 & 250 & 500 & 1000 & 500 \\
\hline $9 a$ & $\mathrm{H}$ & $\mathrm{H}$ & 200 & 500 & 200 & 250 & $>1000$ & 500 \\
\hline $9 b$ & $\mathrm{H}$ & $\mathrm{CH}_{3}$ & 50 & 100 & 250 & 500 & 500 & 250 \\
\hline $9 \mathrm{c}$ & $\mathrm{H}$ & $\mathrm{OCH}_{3}$ & 200 & 200 & 500 & 250 & 500 & 1000 \\
\hline 9d & $\mathrm{OCH}_{3}$ & $\mathrm{H}$ & 250 & 500 & 250 & 200 & 250 & 250 \\
\hline $9 e$ & $\mathrm{OCH}_{3}$ & $\mathrm{CH}_{3}$ & 100 & 250 & 200 & 500 & 250 & 500 \\
\hline 9f & $\mathrm{OCH}_{3}$ & $\mathrm{OCH}_{3}$ & 250 & 500 & 500 & 500 & 500 & 250 \\
\hline \multicolumn{3}{|c|}{ Ampicillin } & 250 & 250 & 100 & 100 & - & - \\
\hline \multicolumn{3}{|c|}{ Nystatin } & - & - & - & - & 100 & 100 \\
\hline \multicolumn{3}{|c|}{ Griseofulvin } & - & - & - & - & 500 & 100 \\
\hline
\end{tabular}

Compounds 3e, $\mathbf{7 b}, \mathbf{8 b}, \mathbf{8 d}(\mathrm{MIC}=100 \mu \mathrm{g} / \mathrm{mL})$ were found to be equipotent against gram negative bacteria Escherichia coli as compared to ampicillin (MIC $=100 \mu \mathrm{g} / \mathrm{mL}$ ) while on the other hand only one compound $7 \mathrm{e}(\mathrm{MIC}=100 \mu \mathrm{g} / \mathrm{mL})$ showed equipotent activity against gram negative bacteria Salmonella typhi as compared to ampicillin (MIC $=100$ $\mu \mathrm{g} / \mathrm{mL})$.

Compounds $\mathbf{3 b}$ and $\mathbf{7 b}(\mathrm{MIC}=100 \mu \mathrm{g} / \mathrm{mL})$ were the only candidates which showed equal activity against anti fungal strain Candida albicans and Aspergillus niger respectively as compared to nystatin ( $\mathrm{MIC}=100 \mu \mathrm{g} / \mathrm{mL}$ ). Compounds 8a, 8c $(\mathrm{MIC}=200 \mu \mathrm{g} / \mathrm{mL}$ ) and 3d, 7c, 8b, 9d, 9e $(\mathrm{MIC}=250 \mu \mathrm{g} / \mathrm{mL})$ showed more activity as compared to grisofluvin (MIC $=$ $500 \mu \mathrm{g} / \mathrm{mL}$ ) against Candida albicans. 
Interestingly, pyrazolyl acrolyl coumarin $3 \mathbf{b}(\mathrm{MIC}=250$ and $200 \mu \mathrm{g} / \mathrm{mL})$ after bypyridyl ring formation in $\mathbf{7 b}$ and $\mathbf{9 b}(\mathrm{MIC}=50$ and $100 \mu \mathrm{g} / \mathrm{mL})$ found to possess increased potency against Staphylococcus aureus and Bacillus subtilis respectively.

A general observation was made from the activity data (Table 1) that compounds 7a-c, 8a-c and 9a-c having no substitution in coumarin ring were more potent than their analogous having methoxyl substitution in most of the cases against all the bacterial strains. Compounds 9a-f bearing 4-pyridyl ring showed poor activity compared to standard drugs against gram negative bacterial strains. Compounds $\mathbf{7 b}$ and $\mathbf{8 b}$, with $\mathrm{R}_{1}=\mathrm{CH}_{3}$ showed better activity than their analogous against all the bacterial strains while $9 \mathrm{~b}$ with $\mathrm{R}_{1}=\mathrm{CH}_{3}$ showed better activity against gram positive bacterial strains only.

\section{EXPERIMENTAL}

\section{1. Chemistry}

All reactions were performed with commercially available reagents and they were used without further purification. Organic solvents were purified by standard methods and stored over molecular sieves. All reactions were monitored by thin-layer chromatography (TLC, on aluminium plates coated with silica gel $60 \mathrm{~F}_{254}, 0.25 \mathrm{~mm}$ thickness, Merck) and detection of the components was made by exposure to UV light. Melting points were determined in open capillaries and are uncorrected. Infrared spectra were recorded on Shimadzu FTIR 8401 spectrophotometer using potassium bromide pellets in the range 4,000-400 $\mathrm{cm}^{-1}$ and frequencies of only characteristic peaks are expressed in $\mathrm{cm}^{-1} .{ }^{1} \mathrm{H}$ and ${ }^{13} \mathrm{C}$ NMR spectra were recorded in $\mathrm{CDCl}_{3}$ on a Bruker Avance $400(\mathrm{MHz})$ spectrometer (Bruker Scientific Corporation Ltd., Switzerland) using TMS signal as an internal standard at $400 \mathrm{MHz}$ and 100 $\mathrm{MHz}$ respectively. Chemical shifts are reported in parts per million (ppm). The coupling constants $(\mathrm{J})$ are given in Hertz $(\mathrm{Hz})$. Mass spectrum of one representative compound was scanned on a Shimadzu QP 2010 spectrometer (Shimadzu, Tokyo, Japan). The compounds were purified by column chromatography using silica gel (60-120 mesh). Reference drugs ampicillin, griseofulvin, nystatin were of commercial grade.

Starting precursors 3-acetyl coumarins 1 [25], pyrazole aldehydes 2 [26], pyridoyl methyl pyridinium iodide salts $\mathbf{4 , 5}$ and $\mathbf{6}$ [27] were prepared using the reported procedures.

\section{1. 1. General procedure for the synthesis of 3-[3-(1-phenyl-3-aryl-1H-pyrazol-4-yl) acryloyl]coumarins 3a-f.}

In a $100 \mathrm{~mL}$ round bottom flask fitted with a reflux condenser, a solution of appropriate 3-acetyl coumarin, $0.01 \mathrm{~mol}$ (1a and $1 \mathrm{~b}: 0.5 \mathrm{~g}$ ), 1-phenyl-3-aryl- $1 H$-pyrazole-4-carbaldehyde (pyrazole aldehyde), $0.01 \mathrm{~mol}$ (2a:0.65g, 2b:0.69g, 2c:0.73g, 2d:0.56g, 2e:0.6g, 2f:0.63g) and catalytic amount of piperidine were taken in ethanol $(30 \mathrm{~mL})$. The reaction mixture was refluxed in water bath for 3 hours. The pyrazolyl acrolyl coumarin was formed, which was filtered out and washed with cold ethanol and dried. It was recrystallized from chloroform.

\section{3-[3-(1,3-diphenyl-1H-pyrazol-4-yl)acryloyl]coumarin (3a):}

Yield: 86\%; m.p. $234^{\circ} \mathrm{C}$; IR $\left(\mathrm{KBr}, v_{\max }, \mathrm{cm}^{-1}\right): 1720(\mathrm{C}=\mathrm{O} \delta$-lactone stretching), $1659(\mathrm{C}=\mathrm{O}$ ketone stretching), 1605 and 1535 (aromatic $\mathrm{C}=\mathrm{C}$ and $\mathrm{C}=\mathrm{N}$ stretchings), $756 \& 687(\mathrm{C}-\mathrm{H}$ bending of mono substituted benzene), 3063 (aromatic $\mathrm{C}-\mathrm{H}$ stretching); ${ }^{1} \mathrm{H}$ NMR $(400 \mathrm{MHz}$, $\left.\mathrm{CDCl}_{3}, \delta\right): 7.35-8.01\left(16 \mathrm{H}, \mathrm{m}, 14 \mathrm{Ar}-\mathrm{H}\right.$ and $\left.\mathrm{C}_{2}{ }^{\prime}-\mathrm{H}, \mathrm{C}_{3}{ }^{\prime}-\mathrm{H}\right), 8.47\left(1 \mathrm{H}, \mathrm{s}, \mathrm{C}_{5}{ }^{\prime}-\mathrm{H}\right), 8.60\left(1 \mathrm{H}, \mathrm{s}, \mathrm{C}_{4}{ }^{-}\right.$ $\mathrm{H}) ;{ }^{13} \mathrm{C}$ NMR $\left(100 \mathrm{MHz}, \mathrm{CDCl}_{3}, \delta\right)$ : $119.69\left(\mathrm{C}_{2}{ }^{\prime}\right), 118.41\left(\mathrm{C}_{10}\right), 118.63\left(\mathrm{C}_{4}{ }^{\prime}\right), 119.49\left(\mathrm{C}_{8}\right)$, 
119.79( $\left(\mathrm{C}_{\mathrm{b}}{ }^{\prime}, \mathrm{C}_{\mathrm{f}}{ }^{\prime}\right), 123.18\left(\mathrm{C}_{\mathrm{d}}{ }^{\prime}\right), 125.00\left(\mathrm{C}_{3}\right), 125.27\left(\mathrm{C}_{6}\right), 127.10\left(\mathrm{C}_{5}{ }^{\prime}\right), 127.34\left(\mathrm{C}_{\mathrm{c}}, \mathrm{C}_{\mathrm{e}}\right), 128.01\left(\mathrm{C}_{\mathrm{b}}\right.$, $\left.\mathrm{C}_{\mathrm{f}}\right), \quad 128.88\left(\mathrm{C}_{\mathrm{d}}\right), \quad 129.32\left(\mathrm{C}_{\mathrm{c}}{ }^{\prime}, \quad \mathrm{C}_{\mathrm{e}}{ }^{\prime}\right), \quad 129.70\left(\mathrm{C}_{5}\right), \quad 130.97\left(\mathrm{C}_{7}\right), \quad 134.20\left(\mathrm{C}_{\mathrm{a}}\right), \quad 135.90\left(\mathrm{C}_{3}{ }^{\prime}\right)$, 139.40 $\left(\mathrm{C}_{\mathrm{a}}{ }^{\prime}\right), 148.14\left(\mathrm{C}_{4}\right), 154.38\left(\mathrm{C}_{9}\right), 155.20\left(\mathrm{C}_{2}\right), 159.47\left(\mathrm{C}_{8}{ }^{\prime}\right), 185.30\left(\mathrm{C}_{1}{ }^{\prime}\right)$; Anal. Calcd. for $\mathrm{C}_{27} \mathrm{H}_{18} \mathrm{~N}_{2} \mathrm{O}_{3}$ C, 77.50; H, 4.34; N, 6.69\%. Found: C, 77.43; H, 4.39; N, 6.61\%.

\section{3-[3-(1-phenyl-3-p-tolyl-1H-pyrazol-4-yl)acryloyl]coumarin (3b):}

Yield: $81 \%$; m.p. $208^{\circ} \mathrm{C}$; IR $\left(\mathrm{KBr}, v_{\max }, \mathrm{cm}^{-1}\right): 1705(\mathrm{C}=\mathrm{O} \delta$-lactone stretching), $1659(\mathrm{C}=\mathrm{O}$ ketone stretching), 1605 and 1528 (aromatic $\mathrm{C}=\mathrm{C}$ and $\mathrm{C}=\mathrm{N}$ stretchings), $764 \& 679(\mathrm{C}-\mathrm{H}$ bending of mono substituted benzene), 3024 (aromatic $\mathrm{C}-\mathrm{H}$ stretching); ${ }^{1} \mathrm{H}$ NMR $(400 \mathrm{MHz}$, $\left.\mathrm{CDCl}_{3}, \delta\right): 2.45\left(3 \mathrm{H}, \mathrm{s}, \mathrm{C}_{\mathrm{d}}-\mathrm{CH}_{3}\right), 7.32-8.01\left(15 \mathrm{H}, \mathrm{m}, 13 \mathrm{Ar}-\mathrm{H}\right.$ and $\left.\mathrm{C}_{2}{ }^{\prime}-\mathrm{H}, \mathrm{C}_{3}{ }^{\prime}-\mathrm{H}\right), 8.46(1 \mathrm{H}, \mathrm{s}$, $\left.\mathrm{C}_{5}{ }^{\prime}-\mathrm{H}\right), 8.60\left(1 \mathrm{H}, \mathrm{s}, \mathrm{C}_{4}-\mathrm{H}\right) ;{ }^{13} \mathrm{C} \mathrm{NMR}\left(100 \mathrm{MHz}, \mathrm{CDCl}_{3}, \delta\right): 21.38\left(\mathrm{C}_{\mathrm{d}}{ }^{\prime}-\mathrm{CH}_{3}\right), 116.68\left(\mathrm{C}_{2}{ }^{\prime}\right)$, 118.35 $\left(\mathrm{C}_{10}\right), 118.65\left(\mathrm{C}_{4}{ }^{\prime}\right), 119.46\left(\mathrm{C}_{8}\right), 123.01\left(\mathrm{C}_{\mathrm{b}}{ }^{\prime}, \mathrm{C}_{\mathrm{f}}{ }^{\prime}\right), 124.97\left(\mathrm{C}_{\mathrm{d}}{ }^{\prime}\right), 125.34\left(\mathrm{C}_{3}\right), 126.94\left(\mathrm{C}_{6}\right)$, 127.25( $\left(\mathrm{C}_{\mathrm{a}}\right), \quad 128.74\left(\mathrm{C}_{\mathrm{c}}, \mathrm{C}_{\mathrm{e}}\right), \quad 129.24\left(\mathrm{C}_{\mathrm{d}}\right), \quad 129.50\left(\mathrm{C}_{5^{\prime}}\right), \quad 129.56\left(\mathrm{C}_{\mathrm{b}}, \mathrm{C}_{\mathrm{f}}\right), 130.02\left(\mathrm{C}_{\mathrm{c}}, \mathrm{C}_{\mathrm{e}}\right)$, 134.15( $\left.\mathrm{C}_{5}\right), \quad 136.09\left(\mathrm{C}_{7}\right), \quad 138.62\left(\mathrm{C}_{\mathrm{a}}{ }^{\prime}\right), \quad 139.44\left(\mathrm{C}_{\mathrm{a}}\right), \quad 148.05\left(\mathrm{C}_{3}{ }^{\prime}\right), \quad 154.46\left(\mathrm{C}_{4}\right), \quad 155.20\left(\mathrm{C}_{2}\right)$, 159.47( $\left(\mathrm{C}_{8}{ }^{\prime}\right)$, 185.15( $\left(\mathrm{C}_{1}{ }^{\prime}\right)$; Anal. Calcd. for $\mathrm{C}_{28} \mathrm{H}_{20} \mathrm{~N}_{2} \mathrm{O}_{3} \mathrm{C}, 77.76 ; \mathrm{H}, 4.66 ; \mathrm{N}, 6.48 \%$. Found: C, 77.84; H, 4.61; N, 6.40\%.

\section{3-[3-\{3-(4-methoxyphenyl)-1-phenyl-1H-pyrazol-4-yl\}acryloyl]coumarin (3c):}

Yield: $84 \%$; m.p. $180^{\circ} \mathrm{C}$; IR $\left(\mathrm{KBr}, v_{\max }, \mathrm{cm}^{-1}\right): 1736(\mathrm{C}=\mathrm{O} \delta$-lactone stretching $), 1666(\mathrm{C}=\mathrm{O}$ ketone stretching), 1612 and 1528 (aromatic $\mathrm{C}=\mathrm{C}$ and $\mathrm{C}=\mathrm{N}$ stretchings), $787 \& 679(\mathrm{C}-\mathrm{H}$ bending of mono substituted benzene), 3040 (aromatic $\mathrm{C}-\mathrm{H}$ stretching); ${ }^{1} \mathrm{H}$ NMR $(400 \mathrm{MHz}$, $\left.\mathrm{CDCl}_{3}, \delta\right): 3.90\left(3 \mathrm{H}, \mathrm{s}, \mathrm{C}_{\mathrm{d}}-\mathrm{OCH}_{3}\right), 7.04-8.00\left(15 \mathrm{H}, \mathrm{m}, 13 \mathrm{Ar}-\mathrm{H}\right.$ and $\left.\mathrm{C}_{2}{ }^{\prime}-\mathrm{H}, \mathrm{C}_{3}{ }^{\prime}-\mathrm{H}\right), 8.45(1 \mathrm{H}$,

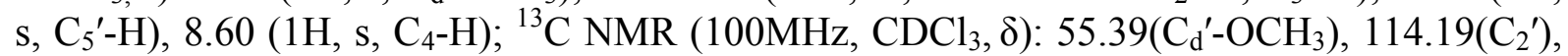
$114.29\left(\mathrm{C}_{\mathrm{c}}, \mathrm{C}_{\mathrm{e}}\right), \quad 116.65\left(\mathrm{C}_{8}\right), \quad 118.21\left(\mathrm{C}_{10}\right), \quad 118.63\left(\mathrm{C}_{4}{ }^{\prime}\right), \quad 119.68\left(\mathrm{C}_{\mathrm{b}}{ }^{\prime}, \mathrm{C}_{\mathrm{f}}{ }^{\prime}\right), \quad 122.95\left(\mathrm{C}_{\mathrm{d}}{ }^{\prime}\right)$, 124.65( $\left(\mathrm{C}_{\mathrm{a}}\right), 124.96\left(\mathrm{C}_{3}\right), 125.31\left(\mathrm{C}_{6}\right), 126.94\left(\mathrm{C}_{5}{ }^{\prime}\right), 127.21\left(\mathrm{C}_{\mathrm{c}}{ }^{\prime}, \mathrm{C}_{\mathrm{e}}{ }^{\prime}\right), 127.89\left(\mathrm{C}_{\mathrm{d}}\right), 129.54\left(\mathrm{C}_{\mathrm{b}}\right.$, $\left.\mathrm{C}_{\mathrm{f}}\right), 130.27\left(\mathrm{C}_{5}\right), 134.14\left(\mathrm{C}_{7}\right), 136.08\left(\mathrm{C}_{3}{ }^{\prime}\right), 139.40\left(\mathrm{C}_{\mathrm{a}}{ }^{\prime}\right), 148.02\left(\mathrm{C}_{4}\right), 154.17\left(\mathrm{C}_{9}\right), 155.17\left(\mathrm{C}_{2}\right)$, 159.44 $\left(\mathrm{C}_{8}{ }^{\prime}\right)$, 160.11 $\left(\mathrm{C}_{\mathrm{d}^{\prime}}{ }^{\prime}\right) 185.05\left(\mathrm{C}_{1}{ }^{\prime}\right)$; Anal. Calcd. for $\mathrm{C}_{28} \mathrm{H}_{20} \mathrm{~N}_{2} \mathrm{O}_{4} \mathrm{C}, 74.99 ; \mathrm{H}, 4.50$; $\mathrm{N}$, 6.25\%. Found: C, 74.90; H, 4.41; N, 6.32\%.

\section{8-methoxy-3-[3-(1,3-diphenyl-1H-pyrazol-4-yl)acryloyl]coumarin (3d):}

Yield: $89 \%$; m.p. $220^{\circ} \mathrm{C}$; IR $\left(\mathrm{KBr}, v_{\max }, \mathrm{cm}^{-1}\right): 1720(\mathrm{C}=\mathrm{O} \delta$-lactone stretching), $1661(\mathrm{C}=\mathrm{O}$ ketone stretching), 1610 and 1535 (aromatic $\mathrm{C}=\mathrm{C}$ and $\mathrm{C}=\mathrm{N}$ stretchings), $780 \& 687$ (C-H bending of mono substituted benzene), 3046 (aromatic $\mathrm{C}-\mathrm{H}$ stretching); ${ }^{1} \mathrm{H}$ NMR $(400 \mathrm{MHz}$, $\left.\mathrm{CDCl}_{3}, \delta\right): 4.02\left(3 \mathrm{H}, \mathrm{s}, \mathrm{C}_{8}-\mathrm{OCH}_{3}\right), 7.19-8.01\left(15 \mathrm{H}, \mathrm{m}, 13 \mathrm{Ar}-\mathrm{H}\right.$ and $\left.\mathrm{C}_{2}{ }^{\prime}-\mathrm{H}, \mathrm{C}_{3}{ }^{\prime}-\mathrm{H}\right), 8.48(1 \mathrm{H}, \mathrm{s}$, $\left.\mathrm{C}_{5}{ }^{\prime}-\mathrm{H}\right), 8.58\left(1 \mathrm{H}, \mathrm{s}, \mathrm{C}_{4}-\mathrm{H}\right) ;{ }^{13} \mathrm{C}$ NMR $\left(100 \mathrm{MHz}, \mathrm{CDCl}_{3}, \delta\right): 55.39\left(\mathrm{C}_{\mathrm{d}}{ }^{\prime}-\mathrm{OCH}_{3}\right), 116.00 .19\left(\mathrm{C}_{2}{ }^{\prime}\right)$, 118.40 $\left(\mathrm{C}_{10}\right), 119.35\left(\mathrm{C}_{4}{ }^{\prime}\right), 121.30\left(\mathrm{C}_{7}\right), 122.10\left(\mathrm{C}_{\mathrm{b}}{ }^{\prime}, \mathrm{C}_{\mathrm{f}}{ }^{\prime}\right), 122.97\left(\mathrm{C}_{6}\right), 125.00\left(\mathrm{C}_{\mathrm{d}}{ }^{\prime}\right), 125.45\left(\mathrm{C}_{5}{ }^{\prime}\right)$, 125.96 $\left(\mathrm{C}_{3}\right), 126.87\left(\mathrm{C}_{5}\right), 127.29\left(\mathrm{C}_{\mathrm{c}}, \mathrm{C}_{\mathrm{e}}\right), 128.65\left(\mathrm{C}_{\mathrm{a}}\right), 129.00\left(\mathrm{C}_{\mathrm{b}}, \mathrm{C}_{\mathrm{f}}\right), 129.89\left(\mathrm{C}_{\mathrm{d}}\right), 135.45\left(\mathrm{C}_{\mathrm{c}}{ }^{\prime}\right.$, $\left.\mathrm{C}_{\mathrm{e}}{ }^{\prime}\right), 138.50\left(\mathrm{C}_{9}\right), 140.65\left(\mathrm{C}_{8}\right), 146.56\left(\mathrm{C}_{\mathrm{a}}{ }^{\prime}\right), 147.68\left(\mathrm{C}_{3}{ }^{\prime}\right), 148.52\left(\mathrm{C}_{4}\right), 155.42\left(\mathrm{C}_{8}{ }^{\prime}\right), 158.17\left(\mathrm{C}_{2}\right)$, $185.09\left(\mathrm{C}_{1}{ }^{\prime}\right)$; Anal. Calcd. for $\mathrm{C}_{28} \mathrm{H}_{20} \mathrm{~N}_{2} \mathrm{O}_{4} \mathrm{C}, 74.99 ; \mathrm{H}, 4.50 ; \mathrm{N}, 6.25 \%$. Found: $\mathrm{C}, 74.89 ; \mathrm{H}$, $4.44 ; \mathrm{N}, 6.30 \%$.

\section{8-methoxy-3-[3-(1-phenyl-3-p-tolyl-1H-pyrazol-4-yl)acryloyl]coumarin (3e):}

Yield: $91 \%$; m.p. $160^{\circ} \mathrm{C}$; IR $\left(\mathrm{KBr}, v_{\max }, \mathrm{cm}^{-1}\right): 1728(\mathrm{C}=\mathrm{O} \delta$-lactone stretching), $1651(\mathrm{C}=\mathrm{O}$ ketone stretching), 1605 and 1574 (aromatic $\mathrm{C}=\mathrm{C}$ and $\mathrm{C}=\mathrm{N}$ stretchings), $779 \& 680(\mathrm{C}-\mathrm{H}$ bending of mono substituted benzene), 3056 (aromatic $\mathrm{C}-\mathrm{H}$ stretching); ${ }^{1} \mathrm{H}$ NMR $(400 \mathrm{MHz}$, $\left.\mathrm{CDCl}_{3}, \delta\right): 2.45\left(3 \mathrm{H}, \mathrm{s}, \mathrm{C}_{\mathrm{d}}-\mathrm{CH}_{3}\right), 4.02\left(3 \mathrm{H}, \mathrm{s}, \mathrm{C}_{8}-\mathrm{OCH}_{3}\right), 7.19-8.01\left(14 \mathrm{H}, \mathrm{m}, 12 \mathrm{Ar}-\mathrm{H}\right.$ and $\mathrm{C}_{2}{ }^{\prime}-$ $\left.\mathrm{H}, \mathrm{C}_{3}{ }^{\prime}-\mathrm{H}\right), 8.46(1 \mathrm{H}, \mathrm{s}, \mathrm{Py}-\mathrm{H}), 8.58\left(1 \mathrm{H}, \mathrm{s}, \mathrm{C}_{4}-\mathrm{H}\right) ;{ }^{13} \mathrm{C} \mathrm{NMR}\left(100 \mathrm{MHz}, \mathrm{CDCl}_{3}, \delta\right): 21.37\left(\mathrm{C}_{\mathrm{d}}{ }^{\prime}-\right.$ $\left.\mathrm{CH}_{3}\right)$, 56.37 $\left(\mathrm{C}_{8}-\mathrm{OCH}_{3}\right), 115.89\left(\mathrm{C}_{2}{ }^{\prime}\right), 118.35\left(\mathrm{C}_{4}{ }^{\prime}\right), 119.27\left(\mathrm{C}_{3}\right), 121.20\left(\mathrm{C}_{7}\right), 121.35\left(\mathrm{C}_{\mathrm{b}}{ }^{\prime}, \mathrm{C}_{\mathrm{f}}{ }^{\prime}\right)$, 
123.02 $\left(\mathrm{C}_{6}\right), 124.80\left(\mathrm{C}_{\mathrm{d}}\right), 125.49\left(\mathrm{C}_{\mathrm{a}}\right), 127.02\left(\mathrm{C}_{5}\right), 127.23(\mathrm{Cc}, \mathrm{Ce}), 128.86\left(\mathrm{C}_{5}{ }^{\prime}\right), 129.24\left(\mathrm{C}_{\mathrm{d}}{ }^{\prime}\right)$, $130.97\left(\mathrm{C}_{\mathrm{b}}, \quad \mathrm{C}_{\mathrm{f}}\right), \quad 136.10\left(\mathrm{C}_{\mathrm{c}}{ }^{\prime}, \quad \mathrm{C}_{\mathrm{e}}{ }^{\prime}\right), \quad 138.60\left(\mathrm{C}_{9}\right), \quad 139.46\left(\mathrm{C}_{8}\right), \quad 147.06\left(\mathrm{C}_{\mathrm{a}}{ }^{\prime}\right), \quad 147.71\left(\mathrm{C}_{3}{ }^{\prime}\right)$, 148.28 $\left(\mathrm{C}_{4}\right), 154.47\left(\mathrm{C}_{8}{ }^{\prime}\right), 158.92\left(\mathrm{C}_{2}\right), 185.46\left(\mathrm{C}_{1}{ }^{\prime}\right)$; Anal. Calcd. for $\mathrm{C}_{29} \mathrm{H}_{22} \mathrm{~N}_{2} \mathrm{O}_{4} \mathrm{C}, 75.31 ; \mathrm{H}$, 4.79 ; N, 6.06\%. Found: C, 75.40; H, 4.72; N, 6.15\%.

8-methoxy-3-[3-\{3-(4-methoxyphenyl)-1-phenyl-1H-pyrazol-4-yl\}acryloyl]coumarin (3f): Yield: $90 \%$; m.p. $188^{\circ} \mathrm{C}$; IR $\left(\mathrm{KBr}, v_{\max }, \mathrm{cm}^{-1}\right): 1720(\mathrm{C}=\mathrm{O} \delta$-lactone stretching), $1666(\mathrm{C}=\mathrm{O}$ ketone stretching), 1605 and 1528 (aromatic $\mathrm{C}=\mathrm{C}$ and $\mathrm{C}=\mathrm{N}$ stretchings), $787 \& 687$ (C-H bending of mono substituted benzene), 3060 (aromatic $\mathrm{C}-\mathrm{H}$ stretching); ${ }^{1} \mathrm{H}$ NMR $(400 \mathrm{MHz}$, $\left.\mathrm{CDCl}_{3}, \delta\right): 3.90\left(3 \mathrm{H}, \mathrm{s}, \mathrm{C}_{\mathrm{d}}-\mathrm{OCH}_{3}\right), 4.02\left(3 \mathrm{H}, \mathrm{s}, \mathrm{C}_{8}-\mathrm{OCH}_{3}\right), 7.04-8.00(14 \mathrm{H}, \mathrm{m}, 12 \mathrm{Ar}-\mathrm{H}$ and $\left.\mathrm{C}_{2}{ }^{\prime}-\mathrm{H}, \mathrm{C}_{3}{ }^{\prime}-\mathrm{H}\right), 8.45\left(1 \mathrm{H}, \mathrm{s}, \mathrm{C}_{5}{ }^{\prime}-\mathrm{H}\right), 8.58\left(1 \mathrm{H}, \mathrm{s}, \mathrm{C}_{4}-\mathrm{H}\right) ;{ }^{13} \mathrm{C} \mathrm{NMR}\left(100 \mathrm{MHz}, \mathrm{CDCl}_{3}, \delta\right)$ : 55.39 $\left(\mathrm{C}_{\mathrm{d}}{ }^{\prime}-\mathrm{OCH}_{3}\right), \quad 56.57\left(\mathrm{C}_{8}-\mathrm{OCH}_{3}\right), \quad 114.29\left(\mathrm{C}_{2}{ }^{\prime}\right), \quad 115.66\left(\mathrm{C}_{7}\right), \quad 118.22\left(\mathrm{C}_{10}\right), \quad 119.43\left(\mathrm{C}_{4}{ }^{\prime}\right)$, 119.71( $\left(\mathrm{C}_{\mathrm{c}}, \mathrm{C}_{\mathrm{e}}\right), \quad 121.19\left(\mathrm{C}_{\mathrm{b}}{ }^{\prime}, \quad \mathrm{C}_{\mathrm{f}}{ }^{\prime}\right), \quad 122.97\left(\mathrm{C}_{6}\right), \quad 124.67\left(\mathrm{C}_{\mathrm{d}}{ }^{\prime}\right), \quad 125.49\left(\mathrm{C}_{3}\right), \quad 127.00\left(\mathrm{C}_{5}\right)$, 127.20 $\left(\mathrm{C}_{5}{ }^{\prime}\right), \quad 129.67\left(\mathrm{C}_{\mathrm{c}}{ }^{\prime}, \mathrm{C}_{\mathrm{e}}{ }^{\prime}\right), \quad 130.27\left(\mathrm{C}_{\mathrm{b}}, \mathrm{C}_{\mathrm{f}}\right), \quad 136.12\left(\mathrm{C}_{3}{ }^{\prime}\right), \quad 139.44\left(\mathrm{C}_{9}\right), \quad 144.88\left(\mathrm{C}_{8}\right)$, 147.06 $\left(\mathrm{C}_{\mathrm{a}}{ }^{\prime}\right), 148.27\left(\mathrm{C}_{4}\right), 154.21\left(\mathrm{C}_{8}{ }^{\prime}\right), 158.92\left(\mathrm{C}_{2}\right), 160.10\left(\mathrm{C}_{\mathrm{d}}\right), 185.55\left(\mathrm{C}_{1}{ }^{\prime}\right)$; Anal. Calcd. for $\mathrm{C}_{29} \mathrm{H}_{22} \mathrm{~N}_{2} \mathrm{O}_{5} \mathrm{C}, 72.79 ; \mathrm{H}, 4.63 ; \mathrm{N}, 5.85 \%$. Found: C, 72.72; H, 4.55; N, 5.79\%.

\section{3. General procedure for the synthesis of bipyridinyl substituted coumarins (7a-f, 8a-f, and 9a-f).}

In a $100 \mathrm{~mL}$ round bottom flask equipped with a condenser, guard tube and magnetic needle, an appropriate pyridoyl methyl pyridinium iodide salt 4, 5 and $\mathbf{6}, 0.003 \mathrm{~mol}$ (4,5and 6: $1 \mathrm{~g})$ in glacial acetic acid $(15 \mathrm{~mL})$ was taken. To this ammonium acetate, $0.03 \mathrm{~mol}(2.36 \mathrm{~g})$ was added with stirring at room temperature. Then a solution of an appropriate 3-[3-(1,3-diaryl$1 H$-pyrazol-4-yl)acryloyl]coumarin 3a-f, $0.003 \mathrm{~mol}(3 \mathrm{a}: 1.33 \mathrm{~g}, 3 \mathrm{~b}: 1.37 \mathrm{~g}, 3 \mathrm{c}: 1.42 \mathrm{~g}, 3 \mathrm{~d}: 1.42 \mathrm{~g}$, $3 \mathrm{e}: 1.46 \mathrm{~g}, 3 \mathrm{f}: 1.5 \mathrm{~g})$ in glacial acetic acid $(15 \mathrm{~mL})$ was added with stirring at room temperature and the reaction mixture was further stirred for 1 hour at room temperature and then refluxed for 8 hours at $140^{\circ} \mathrm{C}$. It was then allowed to come to room temperature and was poured into ice-cold water $(75 \mathrm{~mL})$. A crude solid obtained was extracted with chloroform $(3 \times 30 \mathrm{~mL})$. The organic layer was washed with $5 \%$ sodium bicarbonate solution $(3 \times 20 \mathrm{~mL})$, water $(2 \times$ $20 \mathrm{~mL}$ ) and dried over anhydrous sodium sulfate.

\section{3-[4-(1,3-Diphenyl-1H-pyrazol-4-yl)-2,2'-bipyridin-6-yl]coumarin (7a):}

Yield: $68 \%$; m.p. $210^{\circ} \mathrm{C}$; IR $\left(\mathrm{KBr}, v_{\max }, \mathrm{cm}^{-1}\right)$ : $1722(\mathrm{C}=\mathrm{O} \delta$-lactone stretching), 1596 and 1474 (aromatic $\mathrm{C}=\mathrm{C}$ and $\mathrm{C}=\mathrm{N}$ stretchings), 3058 (aromatic $\mathrm{C}-\mathrm{H}$ stretching); ${ }^{1} \mathrm{H}$ NMR $\left(400 \mathrm{MHz}, \mathrm{CDCl}_{3}, \delta\right)$ : 7.34-8.47 $\left(19 \mathrm{H}, \mathrm{m}, \mathrm{Ar}-\mathrm{H}\right.$ except $\mathrm{C}_{5}{ }^{\prime}-\mathrm{H}$ and $\left.\mathrm{C}_{6}{ }^{\prime \prime}-\mathrm{H}\right), 8.55(1 \mathrm{H}$, poorly resolved dd, $\left.\mathrm{C}_{6}{ }^{\prime \prime}-\mathrm{H}\right), 8.70\left(1 \mathrm{H}, \mathrm{d}, J=4.0 \mathrm{~Hz}, \mathrm{C}_{5}{ }^{\prime}-\mathrm{H}\right), 8.89\left(1 \mathrm{H}, \mathrm{s}, \mathrm{C}_{4}-\mathrm{H}\right) ;{ }^{13} \mathrm{C} \mathrm{NMR}(100 \mathrm{MHz}$, $\left.\mathrm{CDCl}_{3}, \delta\right): 116.45\left(\mathrm{C}_{5}{ }^{\prime}\right), 119.22\left(\mathrm{C}_{8}\right), 119.59\left(\mathrm{C}_{10}\right), 119.85\left(\mathrm{C}_{\mathrm{b}}, \mathrm{C}_{\mathrm{f}}\right), 120.60\left(\mathrm{C}_{4}{ }^{\prime \prime \prime}\right), 121.26\left(\mathrm{C}_{5}{ }^{\prime \prime}\right)$, 123.23 $\left(\mathrm{C}_{\mathrm{d}}\right), 123.88\left(\mathrm{C}_{6}\right), 124.53\left(\mathrm{C}_{3}{ }^{\prime}\right), 125.61\left(\mathrm{C}_{3}\right), 126.85\left(\mathrm{C}_{5}{ }^{\prime \prime \prime}\right), 127.58\left(\mathrm{C}_{\mathrm{d}}{ }^{\prime}\right), 128.39\left(\mathrm{C}_{\mathrm{b}}{ }^{\prime}, \mathrm{C}_{\mathrm{f}}{ }^{\prime}\right)$, $128.59\left(\mathrm{C}_{5}\right), \quad 128.72\left(\mathrm{C}_{3}{ }^{\prime \prime}\right), \quad 128.83\left(\mathrm{C}_{\mathrm{c}}{ }^{\prime}, \mathrm{C}_{\mathrm{e}}{ }^{\prime}\right), \quad 129.52\left(\mathrm{C}_{\mathrm{c}}, \mathrm{C}_{\mathrm{e}}\right), \quad 132.10\left(\mathrm{C}_{7}\right), \quad 132.58\left(\mathrm{C}_{\mathrm{a}}{ }^{\prime}\right)$, 136.87( $\left.\mathrm{C}_{4}{ }^{\prime \prime}\right), 139.75\left(\mathrm{C}_{\mathrm{a}}\right), 142.46\left(\mathrm{C}_{4}\right), 142.69\left(\mathrm{C}_{3}{ }^{\prime \prime \prime}\right), 149.25\left(\mathrm{C}_{6}{ }^{\prime \prime}\right), 150.95\left(\mathrm{C}_{9}\right), 151.28\left(\mathrm{C}_{2}{ }^{\prime \prime}\right)$, 153.99 $\left(\mathrm{C}_{2}{ }^{\prime}\right), 155.96\left(\mathrm{C}_{4}{ }^{\prime}\right), 156.02\left(\mathrm{C}_{6}{ }^{\prime}\right)$ and $160.04\left(\mathrm{C}_{2}\right)$; Anal. Calcd. for $\mathrm{C}_{34} \mathrm{H}_{22} \mathrm{~N}_{4} \mathrm{O}_{2} \mathrm{C}, 78.75$; H, 4.28; N, 10.80\%. Found: C, 78.70; H, 4.34; N, 10.86\%.

\section{3-[4-(1-Phenyl-3-p-tolyl-1H-pyrazol-4-yl)-2,2'-bipyridin-6-yl]coumarin (7b):}

Yield: $75 \%$; m.p. $182^{\circ} \mathrm{C}$; IR $\left(\mathrm{KBr}, v_{\max }, \mathrm{cm}^{-1}\right)$ : $1722(\mathrm{C}=\mathrm{O} \delta$-lactone stretching $), 1596$ and 1474 (aromatic $\mathrm{C}=\mathrm{C}$ and $\mathrm{C}=\mathrm{N}$ stretchings), 3058 (aromatic $\mathrm{C}-\mathrm{H}$ stretching); ${ }^{1} \mathrm{H} \mathrm{NMR}$ $\left(400 \mathrm{MHz}, \mathrm{CDCl}_{3}, \delta\right): 2.39\left(3 \mathrm{H}, \mathrm{s}, \mathrm{CH}_{3}\right), 7.19-8.46\left(18 \mathrm{H}, \mathrm{m}, \mathrm{Ar}-\mathrm{H}\right.$ except $\mathrm{C}_{5}{ }^{\prime}-\mathrm{H}$ and $\left.\mathrm{C}_{6}{ }^{\prime \prime}-\mathrm{H}\right)$, $8.54\left(1 \mathrm{H}\right.$, poorly resolved dd, $\left.\mathrm{C}_{6}{ }^{\prime \prime}-\mathrm{H}\right), 8.69\left(1 \mathrm{H}, \mathrm{d}, J=4.0 \mathrm{~Hz}, \mathrm{C}_{5}{ }^{\prime}-\mathrm{H}\right), 8.88\left(1 \mathrm{H}, \mathrm{s}, \mathrm{C}_{4}-\mathrm{H}\right) ;{ }^{13} \mathrm{C}$ 
NMR $\left(100 \mathrm{MHz}, \mathrm{CDCl}_{3}, \delta\right): 21.37\left(\mathrm{CH}_{3}\right), 116.41\left(\mathrm{C}_{5}{ }^{\prime}\right), 119.17\left(\mathrm{C}_{8}\right), 119.59\left(\mathrm{C}_{10}\right), 119.76\left(\mathrm{C}_{\mathrm{b}}\right.$, $\left.\mathrm{C}_{\mathrm{f}}\right), \quad 120.48\left(\mathrm{C}_{4}{ }^{\prime \prime \prime}\right), \quad 121.26\left(\mathrm{C}_{5}{ }^{\prime \prime}\right), \quad 123.29\left(\mathrm{C}_{\mathrm{d}}\right), \quad 123.85\left(\mathrm{C}_{6}\right), \quad 124.51\left(\mathrm{C}_{3}{ }^{\prime}\right), \quad 125.67\left(\mathrm{C}_{3}\right)$, 126.73( $\left(\mathrm{C}_{5}{ }^{\prime \prime \prime}\right), 127.56\left(\mathrm{C}_{5}\right), 128.47\left(\mathrm{C}_{\mathrm{c}}{ }^{\prime}, \mathrm{C}_{\mathrm{e}}{ }^{\prime}\right), 128.81\left(\mathrm{C}_{3}{ }^{\prime \prime}\right), 129.25\left(\mathrm{C}_{\mathrm{b}}{ }^{\prime}, \mathrm{C}_{\mathrm{f}}{ }^{\prime}\right), 129.49\left(\mathrm{C}_{\mathrm{c}}, \mathrm{C}_{\mathrm{e}}\right)$, 129.67( $\left(\mathrm{C}_{\mathrm{a}}{ }^{\prime}\right), 132.08\left(\mathrm{C}_{7}\right), 136.83\left(\mathrm{C}_{4}{ }^{\prime \prime}\right), 138.07\left(\mathrm{C}_{\mathrm{d}^{\prime}}\right), 139.78\left(\mathrm{C}_{\mathrm{a}}\right), 142.44\left(\mathrm{C}_{4}\right), 142.90\left(\mathrm{C}_{3}{ }^{\prime \prime \prime}\right)$, 149.24 $\left(\mathrm{C}_{6}{ }^{\prime \prime}\right), 150.93\left(\mathrm{C}_{9}\right), 151.20\left(\mathrm{C}_{2}{ }^{\prime \prime}\right), 153.98\left(\mathrm{C}_{2}{ }^{\prime}\right), 155.92\left(\mathrm{C}_{4}{ }^{\prime}\right), 156.03\left(\mathrm{C}_{6}{ }^{\prime}\right), 160.06\left(\mathrm{C}_{2}\right)$; Anal. Calcd. for $\mathrm{C}_{35} \mathrm{H}_{24} \mathrm{~N}_{4} \mathrm{O}_{2} \mathrm{C}, 78.93 ; \mathrm{H}, 4.54 ; \mathrm{N}, 10.52 \%$. Found: C, 78.97; H, 4.48; N, $10.59 \%$.

3-\{4-[3-(4-Methoxyphenyl)-1-phenyl-1H-pyrazol-4-yl]-2,2'-bipyridin-6-yl\}coumarin (7c): Yield: $65 \%$; m.p. $204^{\circ} \mathrm{C}$; IR $\left(\mathrm{KBr}, v_{\max }, \mathrm{cm}^{-1}\right)$ : $1720(\mathrm{C}=\mathrm{O} \delta$-lactone stretching), 1597 and 1458 (aromatic $\mathrm{C}=\mathrm{C}$ and $\mathrm{C}=\mathrm{N}$ stretchings), 3060 (aromatic $\mathrm{C}-\mathrm{H}$ stretching); ${ }^{1} \mathrm{H}$ NMR $\left(400 \mathrm{MHz}, \mathrm{CDCl}_{3}, \delta\right): 3.84\left(3 \mathrm{H}, \mathrm{s}, \mathrm{OCH}_{3}\right), 6.92-8.45\left(18 \mathrm{H}, \mathrm{m}, \mathrm{Ar}-\mathrm{H}\right.$ except $\mathrm{C}_{5}{ }^{\prime}-\mathrm{H}$ and $\left.\mathrm{C}_{6}{ }^{\prime \prime}-\mathrm{H}\right)$, $8.52\left(1 \mathrm{H}\right.$, poorly resolved dd, $\left.\mathrm{C}_{6}{ }^{\prime \prime}-\mathrm{H}\right), 8.70\left(1 \mathrm{H}, \mathrm{d}, J=4.0 \mathrm{~Hz}, \mathrm{C}_{5}{ }^{\prime}-\mathrm{H}\right), 8.91\left(1 \mathrm{H}, \mathrm{s}, \mathrm{C}_{4}-\mathrm{H}\right) ;{ }^{13} \mathrm{C}$ NMR $\left(100 \mathrm{MHz}, \mathrm{CDCl}_{3}, \delta\right): 55.32\left(\mathrm{OCH}_{3}\right), 114.08\left(\mathrm{C}_{5}{ }^{\prime}\right), 116.41\left(\mathrm{C}_{\mathrm{c}}{ }^{\prime}, \mathrm{C}_{\mathrm{e}}{ }^{\prime}\right), 119.14\left(\mathrm{C}_{8}\right)$, 119.23( $\left(\mathrm{C}_{4}{ }^{\prime \prime \prime}\right), 119.58\left(\mathrm{C}_{3}\right), 119.90\left(\mathrm{C}_{\mathrm{c}}, \mathrm{C}_{\mathrm{f}}\right), 120.22\left(\mathrm{C}_{\mathrm{a}}{ }^{\prime}\right), 121.29\left(\mathrm{C}_{5}{ }^{\prime \prime}\right), 123.23\left(\mathrm{C}_{\mathrm{d}}\right), 123.95\left(\mathrm{C}_{6}\right)$, 124.56 $\left(\mathrm{C}_{3}{ }^{\prime}\right), 125.10\left(\mathrm{C}_{\mathrm{a}}\right), 126.72\left(\mathrm{C}_{5}{ }^{\prime \prime \prime}\right), 127.62\left(\mathrm{C}_{5}\right), 128.91\left(\mathrm{C}_{3}{ }^{\prime \prime}\right), 129.50\left(\mathrm{C}_{\mathrm{c}}, \mathrm{C}_{\mathrm{e}}\right), 129.90\left(\mathrm{C}_{\mathrm{b}^{\prime}}\right.$, $\left.\mathrm{C}_{\mathrm{f}}{ }^{\prime}\right), \quad 132.17\left(\mathrm{C}_{7}\right), \quad 137.03\left(\mathrm{C}_{4}{ }^{\prime \prime}\right), \quad 139.75\left(\mathrm{C}_{3}{ }^{\prime \prime \prime}\right), \quad 142.56\left(\mathrm{C}_{9}\right), \quad 142.66\left(\mathrm{C}_{4}\right), \quad 143.10\left(\mathrm{C}_{2}{ }^{\prime \prime}\right)$, 149.16 $\left(\mathrm{C}_{6}{ }^{\prime \prime}\right)$, $150.92\left(\mathrm{C}_{2}{ }^{\prime}\right), 150.99\left(\mathrm{C}_{\mathrm{d}}{ }^{\prime}\right), 153.99\left(\mathrm{C}_{4}{ }^{\prime}\right), 159.79\left(\mathrm{C}_{6}{ }^{\prime}\right), 160.07\left(\mathrm{C}_{2}\right)$; Anal. Calcd. for $\mathrm{C}_{35} \mathrm{H}_{24} \mathrm{~N}_{4} \mathrm{O}_{3} \mathrm{C}, 76.63 ; \mathrm{H}, 4.41 ; \mathrm{N}, 10.21 \%$. Found: $\mathrm{C}, 76.70 ; \mathrm{H}, 4.35 ; \mathrm{N}, 10.15 \%$.

\section{8-Methoxy-3-[4-(1,3-phenyl-1H-pyrazol-4-yl)-2,2'-bipyridin-6-yl]coumarin (7d):}

Yield: $67 \%$; m.p. $188^{\circ} \mathrm{C}$; IR $\left(\mathrm{KBr}, v_{\max }, \mathrm{cm}^{-1}\right)$ : $1725(\mathrm{C}=\mathrm{O} \delta$-lactone stretching), 1592 and 1470 (aromatic $\mathrm{C}=\mathrm{C}$ and $\mathrm{C}=\mathrm{N}$ stretchings), 3064 (aromatic $\mathrm{C}-\mathrm{H}$ stretching); ${ }^{1} \mathrm{H} \mathrm{NMR}$ $\left(400 \mathrm{MHz}, \mathrm{CDCl}_{3}, \delta\right): 4.00\left(3 \mathrm{H}, \mathrm{s}, \mathrm{OCH}_{3}\right), 7.11-8.44\left(18 \mathrm{H}, \mathrm{m}, \mathrm{Ar}-\mathrm{H}\right.$ except $\mathrm{C}_{5}{ }^{\prime}-\mathrm{H}$ and $\left.\mathrm{C}_{6}{ }^{\prime \prime}-\mathrm{H}\right)$, $8.51\left(1 \mathrm{H}\right.$, poorly resolved dd, $\left.\mathrm{C}_{6}{ }^{\prime \prime}-\mathrm{H}\right), 8.69\left(1 \mathrm{H}, \mathrm{d}, J=4.4 \mathrm{~Hz}, \mathrm{C}_{5}{ }^{\prime}-\mathrm{H}\right), 8.88\left(1 \mathrm{H}, \mathrm{s}, \mathrm{C}_{4}-\mathrm{H}\right) ;{ }^{13} \mathrm{C}$ NMR $\left(100 \mathrm{MHz}, \mathrm{CDCl}_{3}, \delta\right): \quad 56.28\left(\mathrm{OCH}_{3}\right), \quad 113.89\left(\mathrm{C}_{5}{ }^{\prime}\right), \quad 119.21\left(\mathrm{C}_{7}\right), 119.88\left(\mathrm{C}_{\mathrm{b}}, \mathrm{C}_{\mathrm{f}}\right)$, $120.20\left(\mathrm{C}_{10}\right), 120.29\left(\mathrm{C}_{5}{ }^{\prime \prime}\right), 120.55\left(\mathrm{C}_{4}{ }^{\prime \prime \prime}\right), 121.27\left(\mathrm{C}_{6}\right), 123.26\left(\mathrm{C}_{5}\right), 123.89\left(\mathrm{C}_{\mathrm{d}}\right), 124.33\left(\mathrm{C}_{3}{ }^{\prime}\right)$, 125.62 $\left(\mathrm{C}_{3}\right), \quad 126.81\left(\mathrm{C}_{5}{ }^{\prime \prime \prime}\right), \quad 127.63\left(\mathrm{C}_{\mathrm{d}}{ }^{\prime}\right), 128.36\left(\mathrm{C}_{\mathrm{b}^{\prime}}{ }^{\prime}, \mathrm{C}_{\mathrm{f}}{ }^{\prime}\right), 128.56\left(\mathrm{C}_{3}{ }^{\prime \prime}\right), 128.68\left(\mathrm{C}_{\mathrm{c}^{\prime}}{ }^{\prime}, \mathrm{C}_{\mathrm{e}}{ }^{\prime}\right)$, 129.51 $\left(\mathrm{C}_{\mathrm{c}}, \mathrm{C}_{\mathrm{e}}\right), 132.56\left(\mathrm{C}_{\mathrm{a}}{ }^{\prime}\right), 136.98\left(\mathrm{C}_{4}{ }^{\prime \prime}\right), 139.73\left(\mathrm{C}_{\mathrm{a}}\right), 142.67\left(\mathrm{C}_{3}{ }^{\prime \prime \prime}\right), 142.73\left(\mathrm{C}_{4}\right), 143.67\left(\mathrm{C}_{9}\right)$, 146.96 $\left(\mathrm{C}_{8}\right), 149.14\left(\mathrm{C}_{6}{ }^{\prime \prime}\right), 150.92\left(\mathrm{C}_{2}{ }^{\prime \prime}\right), 151.26\left(\mathrm{C}_{2}{ }^{\prime}\right), 155.66\left(\mathrm{C}_{4}{ }^{\prime}\right), 155.83\left(\mathrm{C}_{6}{ }^{\prime}\right), 159.45\left(\mathrm{C}_{2}\right)$; Anal. Calcd. for $\mathrm{C}_{36} \mathrm{H}_{24} \mathrm{~N}_{4} \mathrm{O}_{3} \mathrm{C}, 76.63 ; \mathrm{H}, 4.41 ; \mathrm{N}, 10.21 \%$. Found: C, 76.68; H, 4.48; N, $10.17 \%$.

8-Methoxy-3-[4-(1-phenyl-3-p-tolyl-1H-pyrazol-4-yl)-2,2'-bipyridin-6-yl]coumarin (7e): Yield: $74 \%$; m.p. $226^{\circ} \mathrm{C}$; IR $\left(\mathrm{KBr}, v_{\max }, \mathrm{cm}^{-1}\right): 1728(\mathrm{C}=\mathrm{O} \delta$-lactone stretching), 1582 and 1474 (aromatic $\mathrm{C}=\mathrm{C}$ and $\mathrm{C}=\mathrm{N}$ stretchings), 3060 (aromatic $\mathrm{C}-\mathrm{H}$ stretching); ${ }^{1} \mathrm{H} \mathrm{NMR}$ $\left(400 \mathrm{MHz}, \mathrm{CDCl}_{3}, \delta\right): 2.39\left(3 \mathrm{H}, \mathrm{s}, \mathrm{CH}_{3}\right), 4.04\left(3 \mathrm{H}, \mathrm{s}, \mathrm{OCH}_{3}\right), 7.12-8.45(17 \mathrm{H}, \mathrm{m}, \mathrm{Ar}-\mathrm{H}$ except $\mathrm{C}_{5}{ }^{\prime}-\mathrm{H}$ and $\left.\mathrm{C}_{6}{ }^{\prime \prime}-\mathrm{H}\right), 8.53\left(1 \mathrm{H}\right.$, poorly resolved $\left.\mathrm{dd}, \mathrm{C}_{6}{ }^{\prime \prime}-\mathrm{H}\right), 8.69\left(1 \mathrm{H}, \mathrm{d}, J=4.4 \mathrm{~Hz}, \mathrm{C}_{5}{ }^{\prime}-\mathrm{H}\right)$, $8.86\left(1 \mathrm{H}, \mathrm{s}, \mathrm{C}_{4}-\mathrm{H}\right) ;{ }^{13} \mathrm{C} \mathrm{NMR}\left(100 \mathrm{MHz}, \mathrm{CDCl}_{3}, \delta\right): 21.48\left(\mathrm{CH}_{3}\right), 56.44\left(\mathrm{OCH}_{3}\right), 113.86\left(\mathrm{C}_{5}{ }^{\prime}\right)$, 114.10 $\left(\mathrm{C}_{7}\right), 114.62\left(\mathrm{C}_{10}\right), 115.65\left(\mathrm{C}_{4}{ }^{\prime \prime \prime}\right), 119.18\left(\mathrm{C}_{\mathrm{b}}, \mathrm{C}_{\mathrm{f}}\right), 119.94\left(\mathrm{C}_{5}{ }^{\prime \prime}\right), 120.01\left(\mathrm{C}_{3}\right), 120.22\left(\mathrm{C}_{\mathrm{a}}{ }^{\prime}\right)$, $120.49\left(\mathrm{C}_{6}\right), 120.73\left(\mathrm{C}_{\mathrm{d}}{ }^{\prime}\right), \quad 121.24\left(\mathrm{C}_{5}\right), 123.34\left(\mathrm{C}_{\mathrm{a}}\right), 123.81\left(\mathrm{C}_{3}{ }^{\prime \prime \prime}\right), 124.32\left(\mathrm{C}_{\mathrm{d}}\right), 124.38\left(\mathrm{C}_{3}{ }^{\prime}\right)$, 124.63( $\left.\mathrm{C}_{5}{ }^{\prime \prime \prime}\right), 126.70\left(\mathrm{C}_{\mathrm{c}}, \mathrm{C}_{\mathrm{e}}\right), 127.58\left(\mathrm{C}_{3}{ }^{\prime \prime}\right), 128.45\left(\mathrm{C}_{\mathrm{b}^{\prime}}, \mathrm{C}_{\mathrm{f}}{ }^{\prime}\right), 129.24\left(\mathrm{C}_{\mathrm{c}}, \mathrm{C}_{\mathrm{e}}\right), 129.48\left(\mathrm{C}_{9}\right)$, 136.81( $\left(\mathrm{C}_{4}{ }^{\prime \prime}\right), 138.05\left(\mathrm{C}_{8}\right), 142.54\left(\mathrm{C}_{4}\right), 144.77\left(\mathrm{C}_{2}{ }^{\prime \prime}\right), 146.97\left(\mathrm{C}_{2}{ }^{\prime}\right), 149.23\left(\mathrm{C}_{6}{ }^{\prime \prime}\right)$ 151.26( $\left.\mathrm{C}_{4}{ }^{\prime}\right)$, 155.83( $\left(\mathrm{C}_{6}{ }^{\prime}\right)$, 159.45( $\left.\mathrm{C}_{2}\right)$; Anal. Calcd. for $\mathrm{C}_{36} \mathrm{H}_{26} \mathrm{~N}_{4} \mathrm{O}_{3} \mathrm{C}, 76.85 ; \mathrm{H}, 4.66 ; \mathrm{N}, 9.96 \%$. Found: $\mathrm{C}$, 76.78; H, 4.72; N, 9.91\%. 


\section{8-Methoxy-3-\{4-[3-(4-methoxyphenyl)-1-phenyl-1H-pyrazol-4-yl]-2,2'-bipyridin-6-yl\} coumarin (7f):}

Yield: $70 \%$; m.p. $238^{\circ} \mathrm{C}$; IR $\left(\mathrm{KBr}, v_{\max }, \mathrm{cm}^{-1}\right)$ : $1733(\mathrm{C}=\mathrm{O} \delta$-lactone stretching), 1597 and 1477 (aromatic $\mathrm{C}=\mathrm{C}$ and $\mathrm{C}=\mathrm{N}$ stretchings), 3058 (aromatic $\mathrm{C}-\mathrm{H}$ stretching); ${ }^{1} \mathrm{H} \mathrm{NMR}$ $\left(400 \mathrm{MHz}, \mathrm{CDCl}_{3}, \delta\right): 3.84\left(3 \mathrm{H}, \mathrm{s}, \mathrm{OCH}_{3}\right), 4.02\left(3 \mathrm{H}, \mathrm{s}, . \mathrm{OCH}_{3}\right), 6.91-8.45(17 \mathrm{H}, \mathrm{m}, \mathrm{Ar}-\mathrm{H}$ except $\mathrm{C}_{5}{ }^{\prime}-\mathrm{H}$ and $\left.\mathrm{C}_{6}{ }^{\prime \prime}-\mathrm{H}\right), 8.53\left(1 \mathrm{H}\right.$, poorly resolved dd, $\left.\mathrm{C}_{6}{ }^{\prime \prime}-\mathrm{H}\right), 8.70\left(1 \mathrm{H}, \mathrm{d}, J=4.0 \mathrm{~Hz}, \mathrm{C}_{5}{ }^{\prime}-\right.$ $\mathrm{H}), 8.89\left(1 \mathrm{H}, \mathrm{s}, \mathrm{C}_{4}-\mathrm{H}\right) ;{ }^{13} \mathrm{C} \mathrm{NMR}\left(100 \mathrm{MHz}, \mathrm{CDCl}_{3}, \delta\right): 55.27\left(\mathrm{OCH}_{3}\right), 56.29\left(\mathrm{OCH}_{3}\right)$, 114.06 $\left(\mathrm{C}_{5}{ }^{\prime}\right), \quad 119.15\left(\mathrm{C}_{7}\right), \quad 119.27\left(\mathrm{C}_{10}\right), \quad 119.96\left(\mathrm{C}_{\mathrm{c}^{\prime}}, \mathrm{C}_{\mathrm{e}}{ }^{\prime}\right), \quad 120.22\left(\mathrm{C}_{\mathrm{b}}, \mathrm{C}_{\mathrm{f}}\right), \quad 120.33\left(\mathrm{C}_{4}{ }^{\prime \prime \prime}\right)$, $121.31\left(\mathrm{C}_{5}{ }^{\prime \prime}\right), \quad 123.34\left(\mathrm{C}_{6}\right), 123.90\left(\mathrm{C}_{5}\right), 124.36\left(\mathrm{C}_{\mathrm{d}}\right), 125.08\left(\mathrm{C}_{3}\right), 126.70\left(\mathrm{C}_{3}{ }^{\prime}\right), 127.60\left(\mathrm{C}_{5}{ }^{\prime \prime \prime}\right)$, $129.50\left(\mathrm{C}_{3}{ }^{\prime \prime}\right), \quad 129.89\left(\mathrm{C}_{\mathrm{c}}, \mathrm{C}_{\mathrm{e}}\right), \quad 137.08\left(\mathrm{C}_{\mathrm{b}}{ }^{\prime}, \mathrm{C}_{\mathrm{f}}{ }^{\prime}\right), \quad 139.75\left(\mathrm{C}_{\mathrm{a}}\right), \quad 142.76\left(\mathrm{C}_{4}{ }^{\prime \prime}\right), \quad 143.05\left(\mathrm{C}_{3}{ }^{\prime \prime \prime}\right)$, 143.72( $\left.\mathrm{C}_{9}\right), 146.96\left(\mathrm{C}_{8}\right), 149.02\left(\mathrm{C}_{4}\right), 149.14\left(\mathrm{C}_{6}{ }^{\prime \prime}\right), 150.92\left(\mathrm{C}_{2}{ }^{\prime \prime}\right)$ 150.99 $\left(\mathrm{C}_{2}{ }^{\prime}\right), 151.43\left(\mathrm{C}_{\mathrm{d}}{ }^{\prime}\right)$, 153.83 $\left(\mathrm{C}_{4}{ }^{\prime}\right)$, 159.48( $\left(\mathrm{C}_{6}{ }^{\prime}\right)$, $159.78\left(\mathrm{C}_{2}\right)$; Anal. Calcd. for $\mathrm{C}_{36} \mathrm{H}_{26} \mathrm{~N}_{4} \mathrm{O}_{4} \mathrm{C}, 74.73 ; \mathrm{H}, 4.53 ; \mathrm{N}$, 9.68\%. Found: C, 74.78; H, 4.60; N, 9.75\%

\section{3-[4-(1,3-Diphenyl-1H-pyrazol-4-yl)-2,3'-bipyridin-6-yl]coumarin (8a):}

Yield: $72 \%$; m.p. $184^{\circ} \mathrm{C}$; IR $\left(\mathrm{KBr}, v_{\max }, \mathrm{cm}^{-1}\right)$ : $1720(\mathrm{C}=\mathrm{O} \delta$-lactone stretching), 1590 and 1460 (aromatic $\mathrm{C}=\mathrm{C}$ and $\mathrm{C}=\mathrm{N}$ stretchings), 3060 (aromatic $\mathrm{C}-\mathrm{H}$ stretching); ${ }^{1} \mathrm{H} \mathrm{NMR}$ $\left(400 \mathrm{MHz}, \mathrm{CDCl}_{3}, \delta\right): 2.44$ (3H, singlet, $\left.\mathrm{CH}_{3}\right), 7.24-8.20(16 \mathrm{H}, \mathrm{m}, \mathrm{Ar}-\mathrm{H}), 8.34\left(1 \mathrm{H}, \mathrm{s}, \mathrm{C}_{5}{ }^{\prime \prime \prime}-\mathrm{H}\right)$, $8.60\left(1 \mathrm{H}\right.$, poorly resolved d, $\left.\mathrm{C}_{5}{ }^{\prime}-\mathrm{H}\right), 8.66\left(1 \mathrm{H}\right.$, poorly resolved dd, $\left.\mathrm{C}_{6}{ }^{\prime \prime}-\mathrm{H}\right), 8.98\left(1 \mathrm{H}, \mathrm{s}, \mathrm{C}_{4}-\mathrm{H}\right)$, $9.14\left(1 \mathrm{H}, \mathrm{d}, J=2.0 \mathrm{~Hz}, \mathrm{C}_{2}{ }^{\prime \prime}-\mathrm{H}\right) ;{ }^{13} \mathrm{C} \mathrm{NMR}\left(100 \mathrm{MHz}, \mathrm{CDCl}_{3}, \delta\right): 116.41\left(\mathrm{C}_{5}{ }^{\prime}\right), 119.29\left(\mathrm{C}_{8}\right)$, $119.53\left(\mathrm{C}_{10}\right), 120.19\left(\mathrm{C}_{4}{ }^{\prime \prime \prime}\right), 121.71\left(\mathrm{C}_{\mathrm{b}}, \mathrm{C}_{\mathrm{f}}\right), 123.50\left(\mathrm{C}_{5}{ }^{\prime \prime}\right), 124.68\left(\mathrm{C}_{3}{ }^{\prime}\right), 125.02\left(\mathrm{C}_{3}\right), 127.03\left(\mathrm{C}_{\mathrm{d}}\right)$, 127.41 $\left(\mathrm{C}_{6}\right), \quad 128.69\left(\mathrm{C}_{5}{ }^{\prime \prime \prime}\right), \quad 128.71\left(\mathrm{C}_{\mathrm{d}}{ }^{\prime}\right), 128.87\left(\mathrm{C}_{\mathrm{b}}{ }^{\prime}, \mathrm{C}_{\mathrm{f}}{ }^{\prime}\right), 129.01\left(\mathrm{C}_{5}\right), 129.56\left(\mathrm{C}_{\mathrm{c}^{\prime}}{ }^{\prime}, \mathrm{C}_{\mathrm{e}}{ }^{\prime}\right)$, 132.33 $\left(\mathrm{C}_{\mathrm{c}}, \mathrm{C}_{\mathrm{e}}\right), 132.67\left(\mathrm{C}_{3}{ }^{\prime \prime}\right), 134.21\left(\mathrm{C}_{7}\right), 134.64\left(\mathrm{C}_{\mathrm{a}}{ }^{\prime}\right), 139.62\left(\mathrm{C}_{\mathrm{a}}\right), 140.42\left(\mathrm{C}_{4}\right), 142.50\left(\mathrm{C}_{3}{ }^{\prime \prime \prime}\right)$, 142.86( $\left(\mathrm{C}_{4}{ }^{\prime \prime}\right), 148.41\left(\mathrm{C}_{6}{ }^{\prime \prime}\right), 149.95\left(\mathrm{C}_{2}{ }^{\prime \prime}\right), 151.32\left(\mathrm{C}_{9}\right), 151.74\left(\mathrm{C}_{2}{ }^{\prime}\right), 154.01\left(\mathrm{C}_{4}{ }^{\prime}\right), 154.14\left(\mathrm{C}_{6}{ }^{\prime}\right)$, 160.27 $\left(\mathrm{C}_{2}\right)$; Anal. Calcd. for $\mathrm{C}_{34} \mathrm{H}_{22} \mathrm{~N}_{4} \mathrm{O}_{2} \mathrm{C}, 78.75 ; \mathrm{H}, 4.28 ; \mathrm{N}, 10.80 \%$. Found: $\mathrm{C}, 78.69 ; \mathrm{H}$, $4.36 ; \mathrm{N}, 10.76 \%$.

3-[4-(1-Phenyl-3-p-tolyl-1H-pyrazol-4-yl)-2,3'-bipyridin-6-yl]coumarin (8b):

Yield: $74 \%$; m.p. $220^{\circ} \mathrm{C}$; IR $\left(\mathrm{KBr}, v_{\max }, \mathrm{cm}^{-1}\right)$ : $1720(\mathrm{C}=\mathrm{O} \delta$-lactone stretching), 1590 and 1460 (aromatic $\mathrm{C}=\mathrm{C}$ and $\mathrm{C}=\mathrm{N}$ stretchings), 3060 (aromatic $\mathrm{C}-\mathrm{H}$ stretching); ${ }^{1} \mathrm{H} \mathrm{NMR}$ $\left(400 \mathrm{MHz}, \mathrm{CDCl}_{3}, \delta\right): 2.44$ (3H, singlet, $\left.\mathrm{CH}_{3}\right), 7.24-8.20(16 \mathrm{H}, \mathrm{m}, \mathrm{Ar}-\mathrm{H}), 8.34\left(1 \mathrm{H}, \mathrm{s}, \mathrm{C}_{5}{ }^{\prime \prime \prime}-\mathrm{H}\right)$, $8.60\left(1 \mathrm{H}\right.$, poorly resolved d, $\left.\mathrm{C}_{5}{ }^{\prime}-\mathrm{H}\right), 8.66\left(1 \mathrm{H}\right.$, poorly resolved dd, $\left.\mathrm{C}_{6}{ }^{\prime \prime}-\mathrm{H}\right), 8.98\left(1 \mathrm{H}, \mathrm{s}, \mathrm{C}_{4}-\mathrm{H}\right)$, $9.14\left(1 \mathrm{H}, \mathrm{d}, J=2.0 \mathrm{~Hz}, \mathrm{C}_{2}{ }^{\prime \prime}-\mathrm{H}\right) ;{ }^{13} \mathrm{C} \mathrm{NMR}\left(100 \mathrm{MHz}, \mathrm{CDCl}_{3}, \delta\right): 21.40\left(\mathrm{CH}_{3}\right), 116.41\left(\mathrm{C}_{5}{ }^{\prime}\right)$, $119.24\left(\mathrm{C}_{8}\right), 119.34\left(\mathrm{C}_{\mathrm{b}}, \mathrm{C}_{\mathrm{f}}\right), 119.53\left(\mathrm{C}_{10}\right), 120.06\left(\mathrm{C}_{4}{ }^{\prime \prime \prime}\right), 121.79\left(\mathrm{C}_{5}{ }^{\prime \prime}\right), 123.54\left(\mathrm{C}_{3}{ }^{\prime}\right), 124.68\left(\mathrm{C}_{\mathrm{d}}\right)$, 125.05( $\left(\mathrm{C}_{\mathrm{a}}{ }^{\prime}\right), \quad 126.93\left(\mathrm{C}_{6}\right), \quad 127.41\left(\mathrm{C}_{5}{ }^{\prime \prime \prime}\right), \quad 128.66\left(\mathrm{C}_{5}\right), \quad 129.01\left(\mathrm{C}_{\mathrm{c}}{ }^{\prime}, \mathrm{C}_{\mathrm{e}}{ }^{\prime}\right), \quad 129.36\left(\mathrm{C}_{\mathrm{b}^{\prime}}{ }^{\prime}, \mathrm{C}_{\mathrm{f}}{ }^{\prime}\right)$, $129.54\left(\mathrm{C}_{\mathrm{c}}, \mathrm{C}_{\mathrm{e}}\right), 129.71\left(\mathrm{C}_{3}{ }^{\prime \prime}\right), 132.32\left(\mathrm{C}_{7}\right), 134.72\left(\mathrm{C}_{4}\right), 138.50\left(\mathrm{C}_{\mathrm{d}}{ }^{\prime}\right), 139.64\left(\mathrm{C}_{\mathrm{a}}\right), 142.72\left(\mathrm{C}_{3}{ }^{\prime \prime \prime}\right)$, $142.85\left(\mathrm{C}_{4}{ }^{\prime \prime}\right), 148.36\left(\mathrm{C}_{6}{ }^{\prime \prime}\right), 149.85\left(\mathrm{C}_{2}{ }^{\prime \prime}\right), 151.26\left(\mathrm{C}_{9}\right), 151.72\left(\mathrm{C}_{2}{ }^{\prime}\right), 154.00\left(\mathrm{C}_{4}{ }^{\prime}\right), 154.04\left(\mathrm{C}_{6}{ }^{\prime}\right)$, 160.27( $\left(\mathrm{C}_{2}\right)$; Anal. Calcd. for $\mathrm{C}_{35} \mathrm{H}_{24} \mathrm{~N}_{4} \mathrm{O}_{2} \mathrm{C}, 78.93$; H, 4.54; N, 10.52\%. Found: $\mathrm{C}, 78.86 ; \mathrm{H}$, $4.59 ; \mathrm{N}, 10.47 \%$.

3-\{4-[3-(4-Methoxyphenyl)-1-phenyl-1H-pyrazol-4-yl]-2,3'-bipyridin-6-yl\}coumarin (8c): Yield: 64\%; m.p. $216^{\circ} \mathrm{C}$; IR $\left(\mathrm{KBr}, v_{\max }, \mathrm{cm}^{-1}\right): 1721(\mathrm{C}=\mathrm{O} \delta$-lactone stretching), 1596 and 1456 (aromatic $\mathrm{C}=\mathrm{C}$ and $\mathrm{C}=\mathrm{N}$ stretchings), 3065 (aromatic $\mathrm{C}-\mathrm{H}$ stretching); ${ }^{1} \mathrm{H} \mathrm{NMR}$ $\left(400 \mathrm{MHz}, \mathrm{CDCl}_{3}, \delta\right): 3.88\left(3 \mathrm{H}, \mathrm{s}, \mathrm{OCH}_{3}\right), 6.97-8.23(16 \mathrm{H}, \mathrm{m}, \mathrm{Ar}-\mathrm{H}), 8.34\left(1 \mathrm{H}, \mathrm{s}, \mathrm{C}_{5}{ }^{\prime \prime \prime}-\mathrm{H}\right)$, $8.61\left(1 \mathrm{H}, \mathrm{d}, J=1.2 \mathrm{~Hz}, \mathrm{C}_{5}{ }^{\prime}-\mathrm{H}\right), 8.67\left(1 \mathrm{H}\right.$, poorly resolved dd, $\left.\mathrm{C}_{6}{ }^{\prime \prime}-\mathrm{H}\right), 8.96\left(1 \mathrm{H}, \mathrm{s}, \mathrm{C}_{4}-\mathrm{H}\right)$, $9.19\left(1 \mathrm{H}\right.$, poorly resolved $\left.\mathrm{d}, \mathrm{C}_{2}{ }^{\prime \prime}-\mathrm{H}\right) ;{ }^{13} \mathrm{C} \mathrm{NMR}\left(100 \mathrm{MHz}, \mathrm{CDCl}_{3}, \delta\right): 55.41\left(\mathrm{OCH}_{3}\right)$, 114.17 $\left(\mathrm{C}_{\mathrm{c}}{ }^{\prime}, \quad \mathrm{C}_{\mathrm{e}}{ }^{\prime}\right), \quad 116.45\left(\mathrm{C}_{5}{ }^{\prime}\right), \quad 119.27\left(\mathrm{C}_{8}\right), \quad 119.38\left(\mathrm{C}_{\mathrm{b}}, \mathrm{C}_{\mathrm{f}}\right), \quad 119.95\left(\mathrm{C}_{5}{ }^{\prime \prime}\right), \quad 121.90\left(\mathrm{C}_{3}{ }^{\prime}\right)$, $123.60\left(\mathrm{C}_{\mathrm{d}}\right), 124.71\left(\mathrm{C}_{6}\right), 125.05\left(\mathrm{C}_{10}\right), 125.13\left(\mathrm{C}_{4}{ }^{\prime \prime}\right), 126.12\left(\mathrm{C}_{3}\right), 126.92\left(\mathrm{C}_{5}{ }^{\prime \prime}\right), 127.43\left(\mathrm{C}_{5}\right)$, 
$129.01\left(\mathrm{C}_{\mathrm{c}}, \quad \mathrm{C}_{\mathrm{e}}\right), \quad 129.55\left(\mathrm{C}_{\mathrm{b}}{ }^{\prime}, \quad \mathrm{C}_{\mathrm{f}^{\prime}}\right), \quad 130.01\left(\mathrm{C}_{7}\right), \quad 132.36\left(\mathrm{C}_{4}\right), \quad 134.42\left(\mathrm{C}_{4}{ }^{\prime \prime}\right), \quad 134.80\left(\mathrm{C}_{\mathrm{a}}{ }^{\prime}\right)$, 139.67( $\left(\mathrm{C}_{3}{ }^{\prime \prime}\right), 142.81\left(\mathrm{C}_{\mathrm{a}}\right), 142.89\left(\mathrm{C}_{6}{ }^{\prime \prime}\right), 148.25\left(\mathrm{C}_{2}{ }^{\prime \prime}\right), 149.74\left(\mathrm{C}_{3}{ }^{\prime \prime \prime}\right), 150.99\left(\mathrm{C}_{9}\right), 151.81\left(\mathrm{C}_{\mathrm{d}}{ }^{\prime}\right)$, 154.02 $\left(\mathrm{C}_{2}{ }^{\prime}\right), 154.08\left(\mathrm{C}_{4}{ }^{\prime}\right), 160.03\left(\mathrm{C}_{6}{ }^{\prime}\right), 160.27\left(\mathrm{C}_{2}\right)$; Anal. Calcd. for $\mathrm{C}_{35} \mathrm{H}_{24} \mathrm{~N}_{4} \mathrm{O}_{3} \mathrm{C}, 76.63 ; \mathrm{H}$, 4.41 ; N, 10.21\%. Found: C, 76.58; H, 4.36; N, 10.29\%.

\section{8-Methoxy-3-[4-(1,3-phenyl-1H-pyrazol-4-yl)-2,3'-bipyridin-6-yl]coumarin (8d):}

Yield: $68 \%$; m.p. $240^{\circ} \mathrm{C}$; IR $\left(\mathrm{KBr}, v_{\max }, \mathrm{cm}^{-1}\right): 1713(\mathrm{C}=\mathrm{O} \delta$-lactone stretching), 1597 and 1474 (aromatic $\mathrm{C}=\mathrm{C}$ and $\mathrm{C}=\mathrm{N}$ stretchings), 3062 (aromatic $\mathrm{C}-\mathrm{H}$ stretching); ${ }^{1} \mathrm{H}$ NMR $\left(400 \mathrm{MHz}, \mathrm{CDCl}_{3}, \delta\right): 4.02\left(3 \mathrm{H}, \mathrm{s}, \mathrm{OCH}_{3}\right), 7.14-8.18(16 \mathrm{H}, \mathrm{m}, \mathrm{Ar}-\mathrm{H}), 8.39\left(1 \mathrm{H}, \mathrm{s}, \mathrm{C}_{5}{ }^{\prime \prime \prime}-\mathrm{H}\right)$, $8.62\left(1 \mathrm{H}\right.$, poorly resolved d, $\left.\mathrm{C}_{5}{ }^{\prime}-\mathrm{H}\right), 8.65\left(1 \mathrm{H}\right.$, poorly resolved dd, $\left.\mathrm{C}_{6}{ }^{\prime \prime}-\mathrm{H}\right), 8.96\left(1 \mathrm{H}, \mathrm{s}_{2} \mathrm{C}_{4}-\mathrm{H}\right)$, $9.11\left(1 \mathrm{H}, \mathrm{d}, J=1.8 \mathrm{~Hz}, \mathrm{C}_{2}{ }^{\prime \prime}-\mathrm{H}\right) ;{ }^{13} \mathrm{C} \mathrm{NMR}\left(100 \mathrm{MHz}, \mathrm{CDCl}_{3}, \delta\right): 56.32\left(\mathrm{OCH}_{3}\right), 114.08\left(\mathrm{C}_{7}\right)$, 119.29( $\left.\mathrm{C}_{5}{ }^{\prime}\right), 119.34\left(\mathrm{C}_{\mathrm{c}}, \mathrm{C}_{\mathrm{f}}\right), 120.15\left(\mathrm{C}_{4}{ }^{\prime \prime \prime}\right), 120.42\left(\mathrm{C}_{5}{ }^{\prime \prime}\right), 121.81\left(\mathrm{C}_{3}{ }^{\prime}\right), 123.59\left(\mathrm{C}_{6}\right), 124.51\left(\mathrm{C}_{5}\right)$, 125.15( $\left.\mathrm{C}_{3}\right), \quad 127.02\left(\mathrm{C}_{\mathrm{d}}\right), \quad 127.49\left(\mathrm{C}_{5}^{\prime \prime \prime}\right), \quad 128.67\left(\mathrm{C}_{\mathrm{d}}{ }^{\prime \prime}\right), \quad 128.71\left(\mathrm{C}_{\mathrm{b}}{ }^{\prime}, \mathrm{C}_{\mathrm{f}}{ }^{\prime}\right), \quad 128.87\left(\mathrm{C}_{\mathrm{c}}{ }^{\prime}, \mathrm{C}_{\mathrm{e}}{ }^{\prime}\right)$, $129.56\left(\mathrm{C}_{\mathrm{c}}, \mathrm{C}_{\mathrm{e}}\right), 132.67\left(\mathrm{C}_{3}{ }^{\prime \prime}\right), 134.46\left(\mathrm{C}_{4}{ }^{\prime \prime}\right), 134.79\left(\mathrm{C}_{\mathrm{a}}{ }^{\prime}\right), 136.44\left(\mathrm{C}_{\mathrm{a}}\right), 139.63\left(\mathrm{C}_{3}{ }^{\prime \prime}\right), 142.56\left(\mathrm{C}_{9}\right)$, 143.03 $\left(\mathrm{C}_{4}\right), 143.68\left(\mathrm{C}_{8}\right), 148.17\left(\mathrm{C}_{6}{ }^{\prime \prime}\right), 149.66\left(\mathrm{C}_{2}{ }^{\prime \prime}\right), 151.32\left(\mathrm{C}_{2}{ }^{\prime}\right), 151.77\left(\mathrm{C}_{4}{ }^{\prime}\right), 153.97\left(\mathrm{C}_{6}{ }^{\prime}\right)$, 159.71 $\left(\mathrm{C}_{2}\right)$; Anal. Calcd. for $\mathrm{C}_{35} \mathrm{H}_{24} \mathrm{~N}_{4} \mathrm{O}_{3} \mathrm{C}, 76.63 ; \mathrm{H}, 4.41 ; \mathrm{N}, 10.21 \%$. Found: $\mathrm{C}, 76.56 ; \mathrm{H}$, $4.50 ; \mathrm{N}, 10.15 \%$

8-Methoxy-3-[4-(1-phenyl-3-p-tolyl-1H-pyrazol-4-yl)-2,3-bipyridin-6-yl]coumarin (8e):

Yield: $76 \%$; m.p. $248^{\circ} \mathrm{C}$; IR $\left(\mathrm{KBr}, v_{\max }, \mathrm{cm}^{-1}\right): 1718(\mathrm{C}=\mathrm{O} \delta$-lactone stretching), 1590 and 1470 (aromatic $\mathrm{C}=\mathrm{C}$ and $\mathrm{C}=\mathrm{N}$ stretchings), 3058 (aromatic $\mathrm{C}-\mathrm{H}$ stretching); ${ }^{1} \mathrm{H} \mathrm{NMR}$ $\left(400 \mathrm{MHz}, \mathrm{CDCl}_{3}, \delta\right): 2.43\left(3 \mathrm{H}, \mathrm{s}, \mathrm{CH}_{3}\right), 4.02\left(3 \mathrm{H}, \mathrm{s}, \mathrm{OCH}_{3}\right), 7.14-8.19$ (15H, m, Ar-H), 8.34 $\left(1 \mathrm{H}, \mathrm{s}, \mathrm{C}_{5}{ }^{\prime \prime \prime}-\mathrm{H}\right), 8.61\left(1 \mathrm{H}\right.$, poorly resolved d, $\left.\mathrm{C}_{5}{ }^{\prime}-\mathrm{H}\right), 8.67\left(1 \mathrm{H}\right.$, poorly resolved dd, $\left.\mathrm{C}_{6}{ }^{\prime \prime}-\mathrm{H}\right)$, $8.96\left(1 \mathrm{H}, \mathrm{s}, \mathrm{C}_{4}-\mathrm{H}\right), 9.12\left(1 \mathrm{H}, \mathrm{d}, J=1.2 \mathrm{~Hz}, \mathrm{C}_{2}{ }^{\prime \prime}-\mathrm{H}\right) ;{ }^{13} \mathrm{C} \mathrm{NMR}\left(100 \mathrm{MHz}, \mathrm{CDCl}_{3}, \delta\right)$ : $21.40\left(\mathrm{CH}_{3}\right), \quad 56.30\left(\mathrm{OCH}_{3}\right), \quad 114.02\left(\mathrm{C}_{7}\right), \quad 119.25\left(\mathrm{C}_{5}{ }^{\prime}\right), \quad 119.40\left(\mathrm{C}_{\mathrm{b}}, \quad \mathrm{C}_{\mathrm{f}}\right), \quad 120.07\left(\mathrm{C}_{10}\right)$, 120.17( $\left.\mathrm{C}_{4}{ }^{\prime \prime}\right), 120.38\left(\mathrm{C}_{5}{ }^{\prime \prime}\right), 121.82\left(\mathrm{C}_{3}{ }^{\prime}\right), 123.48\left(\mathrm{C}_{6}\right), 124.49\left(\mathrm{C}_{5}\right), 125.23\left(\mathrm{C}_{3}\right), 126.90\left(\mathrm{C}_{\mathrm{d}}\right)$, 127.46( $\left.\mathrm{C}_{5}{ }^{\prime \prime \prime}\right), 128.65\left(\mathrm{C}_{\mathrm{c}}{ }^{\prime}, \mathrm{C}_{\mathrm{e}}{ }^{\prime}\right), 129.35\left(\mathrm{C}_{\mathrm{b}}{ }^{\prime}, \mathrm{C}_{\mathrm{f}}{ }^{\prime}\right), 129.53\left(\mathrm{C}_{\mathrm{c}}, \mathrm{C}_{\mathrm{e}}\right), 129.72\left(\mathrm{C}_{\mathrm{a}}{ }^{\prime}\right), 134.24\left(\mathrm{C}_{4}{ }^{\prime \prime}\right)$, 134.68( $\left(\mathrm{C}_{3}{ }^{\prime \prime}\right), 138.47\left(\mathrm{C}_{\mathrm{d}^{\prime}}\right), 139.66\left(\mathrm{C}_{\mathrm{a}}\right), 142.71\left(\mathrm{C}_{3}{ }^{\prime \prime \prime}\right), 142.98\left(\mathrm{C}_{4}\right), 143.67\left(\mathrm{C}_{9}\right), 146.95\left(\mathrm{C}_{8}\right)$, 148.45( $\left(\mathrm{C}_{6}{ }^{\prime \prime}\right), 149.94\left(\mathrm{C}_{2}{ }^{\prime \prime}\right), 151.25\left(\mathrm{C}_{2}{ }^{\prime}\right), 151.69\left(\mathrm{C}_{4}{ }^{\prime}\right), 154.05\left(\mathrm{C}_{6}{ }^{\prime}\right), 159.70\left(\mathrm{C}_{2}\right)$; Anal. Calcd. for $\mathrm{C}_{36} \mathrm{H}_{26} \mathrm{~N}_{4} \mathrm{O}_{3} \mathrm{C}, 76.85 ; \mathrm{H}, 4.66$; N, 9.96\%. Found: C, 76.77; H, 4.58; N, 9.88\%.

\section{8-Methoxy-3-\{4-[3-(4-methoxyphenyl)-1-phenyl-1H-pyrazol-4-yl]-2,3'-bipyridin-6-yl\} coumarin $(\mathbf{8 f})$ :}

Yield: $72 \%$; m.p. $250^{\circ} \mathrm{C}$; IR $\left(\mathrm{KBr}, v_{\max }, \mathrm{cm}^{-1}\right)$ : $1720(\mathrm{C}=\mathrm{O} \delta$-lactone stretching $), 1597$ and 1474 (aromatic $\mathrm{C}=\mathrm{C}$ and $\mathrm{C}=\mathrm{N}$ stretchings), 3057 (aromatic $\mathrm{C}-\mathrm{H}$ stretching); ${ }^{1} \mathrm{H}$ NMR $\left(400 \mathrm{MHz}, \mathrm{CDCl}_{3}, \delta\right): 3.87\left(3 \mathrm{H}, \mathrm{s}, \mathrm{CH}_{3}\right), 4.03\left(3 \mathrm{H}, \mathrm{s}, \mathrm{OCH}_{3}\right), 6.96-8.25(15 \mathrm{H}, \mathrm{m}, \mathrm{Ar}-\mathrm{H}), 8.34$ $\left(1 \mathrm{H}, \mathrm{s}, \mathrm{C}_{5}{ }^{\prime \prime \prime}-\mathrm{H}\right), 8.62\left(1 \mathrm{H}\right.$, poorly resolved d, $\left.\mathrm{C}_{5}{ }^{\prime}-\mathrm{H}\right), 8.68\left(1 \mathrm{H}\right.$, poorly resolved dd, $\left.\mathrm{C}_{6}{ }^{\prime \prime}-\mathrm{H}\right)$, $8.96\left(1 \mathrm{H}, \mathrm{s}, \mathrm{C}_{4}-\mathrm{H}\right), 9.21\left(1 \mathrm{H}\right.$, poorly resolved d, $\left.\mathrm{C}_{2}{ }^{\prime \prime}-\mathrm{H}\right) ;{ }^{13} \mathrm{C} \mathrm{NMR}\left(100 \mathrm{MHz}, \mathrm{CDCl}_{3}, \delta\right)$ : 55.38 $\left(\mathrm{OCH}_{3}\right), \quad 56.32\left(\mathrm{OCH}_{3}\right), \quad 114.09\left(\mathrm{C}_{7}\right), 114.15\left(\mathrm{C}_{\mathrm{c}}{ }^{\prime}, \mathrm{C}_{\mathrm{e}}{ }^{\prime}\right), 119.23\left(\mathrm{C}_{5}{ }^{\prime}\right), 119.34\left(\mathrm{C}_{\mathrm{c}}, \mathrm{C}_{\mathrm{f}}\right)$, $119.85\left(\mathrm{C}_{10}\right), 120.16\left(\mathrm{C}_{4}{ }^{\prime \prime \prime}\right), 120.43\left(\mathrm{C}_{5}{ }^{\prime \prime}\right), 122.01\left(\mathrm{C}_{3}{ }^{\prime}\right), 123.78\left(\mathrm{C}_{6}\right), 124.52\left(\mathrm{C}_{5}\right), 125.01\left(\mathrm{C}_{3}\right)$, 125.15( $\left.\mathrm{C}_{\mathrm{a}}\right), \quad 126.90\left(\mathrm{C}_{\mathrm{d}}\right), \quad 127.49\left(\mathrm{C}_{5}{ }^{\prime \prime \prime}\right), \quad 129.53\left(\mathrm{C}_{\mathrm{c}}, \mathrm{C}_{\mathrm{e}}\right), \quad 130.03\left(\mathrm{C}_{\mathrm{b}}{ }^{\prime}, \mathrm{C}_{\mathrm{f}}{ }^{\prime}\right), \quad 134.85\left(\mathrm{C}_{4}{ }^{\prime \prime}\right)$, 135.00 $\left(\mathrm{C}_{3}{ }^{\prime \prime}\right), 139.64\left(\mathrm{C}_{\mathrm{a}}\right), 142.90\left(\mathrm{C}_{3}{ }^{\prime \prime \prime}\right), 143.08\left(\mathrm{C}_{4}\right), 143.69\left(\mathrm{C}_{9}\right), 146.96\left(\mathrm{C}_{8}\right), 147.81\left(\mathrm{C}_{6}{ }^{\prime \prime}\right)$, 149.19( $\left(\mathrm{C}_{2}{ }^{\prime \prime}\right), 150.98\left(\mathrm{C}_{\mathrm{d}}{ }^{\prime}\right), 151.81\left(\mathrm{C}_{2}{ }^{\prime}\right), 153.72\left(\mathrm{C}_{4}{ }^{\prime}\right), 159.69\left(\mathrm{C}_{6}{ }^{\prime}\right), 159.99\left(\mathrm{C}_{2}\right)$; Anal. Calcd. for $\mathrm{C}_{36} \mathrm{H}_{26} \mathrm{~N}_{4} \mathrm{O}_{4} \mathrm{C}, 74.73 ; \mathrm{H}, 4.53 ; \mathrm{N}, 9.68 \%$. Found: $\mathrm{C}, 74.79 ; \mathrm{H}, 4.60 ; \mathrm{N}, 9.60 \%$

\section{3-[4-(1,3-Diphenyl-1H-pyrazol-4-yl)-2,4'-bipyridin-6-yl]coumarin (9a):}

Yield: $62 \%$; m.p. $212^{\circ} \mathrm{C}$; IR $\left(\mathrm{KBr}, v_{\max }, \mathrm{cm}^{-1}\right)$ : $1720(\mathrm{C}=\mathrm{O} \delta$-lactone stretching), 1597 and 1458 (aromatic $\mathrm{C}=\mathrm{C}$ and $\mathrm{C}=\mathrm{N}$ stretchings), 3052 (aromatic $\mathrm{C}-\mathrm{H}$ stretching); ${ }^{1} \mathrm{H}$ NMR 
$\left(400 \mathrm{MHz}, \mathrm{CDCl}_{3}, \delta\right): 2.45\left(3 \mathrm{H}, \mathrm{s}, \mathrm{CH}_{3}\right), 7.26-7.87(16 \mathrm{H}, \mathrm{m}, \mathrm{Ar}-\mathrm{H}), 8.35\left(1 \mathrm{H}, \mathrm{s}, \mathrm{C}_{5}{ }^{\prime \prime \prime}-\mathrm{H}\right), 8.63$ $\left(1 \mathrm{H}\right.$, poorly resolved $\left.\mathrm{d}, \mathrm{C}_{5}{ }^{\prime}-\mathrm{H}\right), 8.72\left(2 \mathrm{H}\right.$, poorly resolved $\mathrm{dd}, \mathrm{C}_{2}{ }^{\prime \prime}-\mathrm{H}$ and $\left.\mathrm{C}_{6}{ }^{\prime \prime}-\mathrm{H}\right), 8.97(1 \mathrm{H}, \mathrm{s}$, $\left.\mathrm{C}_{4}-\mathrm{H}\right) ;{ }^{13} \mathrm{C}$ NMR $\left(100 \mathrm{MHz}, \mathrm{CDCl}_{3}, \delta\right): 116.45\left(\mathrm{C}_{5}{ }^{\prime}\right), 119.29\left(\mathrm{C}_{8}\right), 119.48\left(\mathrm{C}_{10}\right), 119.56\left(\mathrm{C}_{\mathrm{b}}, \mathrm{C}_{\mathrm{f}}\right)$, 120.02 $\left(\mathrm{C}_{3}\right), 121.05\left(\mathrm{C}_{\mathrm{d}}\right), 122.60\left(\mathrm{C}_{6}\right), 124.70\left(\mathrm{C}_{3}{ }^{\prime}\right), 124.96\left(\mathrm{C}_{4}{ }^{\prime \prime \prime}\right), 127.08\left(\mathrm{C}_{3}{ }^{\prime \prime}\right), 127.40\left(\mathrm{C}_{5}{ }^{\prime \prime \prime}\right)$, 128.67 $\left(\mathrm{C}_{\mathrm{d}}{ }^{\prime}\right), \quad 128.73\left(\mathrm{C}_{\mathrm{b}}{ }^{\prime}, \mathrm{C}_{\mathrm{f}}{ }^{\prime}\right), 128.88\left(\mathrm{C}_{5}\right), 129.02\left(\mathrm{C}_{\mathrm{c}^{\prime}}, \mathrm{C}_{\mathrm{e}}{ }^{\prime}\right), 129.58\left(\mathrm{C}_{\mathrm{c}}, \mathrm{C}_{\mathrm{e}}\right), 132.42\left(\mathrm{C}_{7}\right)$, 132.67( $\left(\mathrm{C}_{\mathrm{a}}{ }^{\prime}\right), 139.58\left(\mathrm{C}_{\mathrm{a}}\right), 142.62\left(\mathrm{C}_{4}{ }^{\prime \prime}\right), 142.94\left(\mathrm{C}_{4}\right), 146.35\left(\mathrm{C}_{3}{ }^{\prime \prime \prime}\right), 150.25\left(\mathrm{C}_{2}{ }^{\prime \prime}, \mathrm{C}_{6}{ }^{\prime \prime}\right), 151.30\left(\mathrm{C}_{9}\right)$, 151.88( $\left(\mathrm{C}_{2}{ }^{\prime}\right), 153.91\left(\mathrm{C}_{4}{ }^{\prime}\right), 154.03\left(\mathrm{C}_{6}{ }^{\prime}\right), 160.24\left(\mathrm{C}_{2}\right)$; Anal. Calcd. for $\mathrm{C}_{34} \mathrm{H}_{22} \mathrm{~N}_{4} \mathrm{O}_{2} \mathrm{C}, 78.75 ; \mathrm{H}$, $4.28 ; \mathrm{N}, 10.80 \%$. Found: C, 78.40; H, 4.21; N, 10.86\%.

\section{3-[4-(1-Phenyl-3-p-tolyl-1H-pyrazol-4-yl)-2,4'-bipyridin-6-yl]coumarin (9b):}

Yield: $76 \%$; m.p. $224^{\circ} \mathrm{C}$; IR $\left(\mathrm{KBr}, v_{\max }, \mathrm{cm}^{-1}\right)$ : $1720(\mathrm{C}=\mathrm{O} \delta$-lactone stretching), 1597 and 1458 (aromatic $\mathrm{C}=\mathrm{C}$ and $\mathrm{C}=\mathrm{N}$ stretchings), 3052 (aromatic $\mathrm{C}-\mathrm{H}$ stretching); ${ }^{1} \mathrm{H}$ NMR $\left(400 \mathrm{MHz}, \mathrm{CDCl}_{3}, \delta\right): 2.45\left(3 \mathrm{H}, \mathrm{s}, \mathrm{CH}_{3}\right), 7.26-7.87(16 \mathrm{H}, \mathrm{m}, \mathrm{Ar}-\mathrm{H}), 8.35\left(1 \mathrm{H}, \mathrm{s}, \mathrm{C}_{5}{ }^{\prime \prime \prime}-\mathrm{H}\right), 8.63$ $\left(1 \mathrm{H}\right.$, poorly resolved $\left.\mathrm{d}, \mathrm{C}_{5}{ }^{\prime}-\mathrm{H}\right), 8.72\left(2 \mathrm{H}\right.$, poorly resolved $\mathrm{dd}, \mathrm{C}_{2}{ }^{\prime \prime}-\mathrm{H}$ and $\left.\mathrm{C}_{6}{ }^{\prime \prime}-\mathrm{H}\right), 8.97(1 \mathrm{H}, \mathrm{s}$, $\left.\mathrm{C}_{4}-\mathrm{H}\right) ;{ }^{13} \mathrm{C}$ NMR $\left(100 \mathrm{MHz}, \mathrm{CDCl}_{3}, \delta\right): 21.51\left(\mathrm{CH}_{3}\right), 116.46\left(\mathrm{C}_{5}{ }^{\prime}\right), 119.26\left(\mathrm{C}_{8}\right), 119.51\left(\mathrm{C}_{10}\right)$, $119.62\left(\mathrm{C}_{\mathrm{b}}, \mathrm{C}_{\mathrm{f}}\right), 119.95\left(\mathrm{C}_{4}{ }^{\prime \prime \prime}\right), 121.05\left(\mathrm{C}_{\mathrm{d}}\right), 122.61\left(\mathrm{C}_{6}\right), 124.69\left(\mathrm{C}_{3}{ }^{\prime}\right), 125.06\left(\mathrm{C}_{3}\right), 126.99\left(\mathrm{C}_{3}{ }^{\prime \prime}\right.$, $\left.\mathrm{C}_{5}{ }^{\prime \prime}\right), 127.34\left(\mathrm{C}_{5}{ }^{\prime \prime \prime}\right), 128.70\left(\mathrm{C}_{5}{ }^{\prime}\right), 129.01\left(\mathrm{C}_{\mathrm{c}}{ }^{\prime}, \mathrm{C}_{\mathrm{e}}{ }^{\prime}\right), 129.38\left(\mathrm{C}_{\mathrm{b}}{ }^{\prime}, \mathrm{C}_{\mathrm{f}}{ }^{\prime}\right), 129.55\left(\mathrm{C}_{\mathrm{c}}, \mathrm{C}_{\mathrm{e}}\right), 129.75\left(\mathrm{C}_{\mathrm{a}}{ }^{\prime}\right)$, $132.39\left(\mathrm{C}_{7}\right), 138.49\left(\mathrm{C}_{\mathrm{d}}{ }^{\prime}\right), 139.63\left(\mathrm{C}_{\mathrm{a}}\right), 142.80\left(\mathrm{C}_{4}{ }^{\prime \prime}\right), 142.91\left(\mathrm{C}_{4}\right), 146.28\left(\mathrm{C}_{3}{ }^{\prime \prime}\right), 150.37\left(\mathrm{C}_{2}{ }^{\prime \prime}\right.$, $\left.\mathrm{C}_{6}{ }^{\prime \prime}\right)$, 151.31( $\left.\mathrm{C}_{9}\right), 151.85\left(\mathrm{C}_{2}{ }^{\prime}\right), 153.94\left(\mathrm{C}_{4}{ }^{\prime}\right), 154.04\left(\mathrm{C}_{6}{ }^{\prime}\right), 160.25\left(\mathrm{C}_{2}\right)$; Anal. Calcd. for $\mathrm{C}_{35} \mathrm{H}_{24} \mathrm{~N}_{4} \mathrm{O}_{2}$ C, $78.93 ; \mathrm{H}, 4.54 ; \mathrm{N}, 10.52 \%$. Found: C, 78.87; H, 4.61; N, 10.60\%.

3-\{4-[3-(4-Methoxyphenyl)-1-phenyl-1H-pyrazol-4-yl]-2,4'-bipyridin-6-yl\}coumarin (9c): Yield: $70 \%$; m.p. $210^{\circ} \mathrm{C}$; IR $\left(\mathrm{KBr}, v_{\max }, \mathrm{cm}^{-1}\right): 1718(\mathrm{C}=\mathrm{O} \delta$-lactone stretching), 1595 and 1460 (aromatic $\mathrm{C}=\mathrm{C}$ and $\mathrm{C}=\mathrm{N}$ stretchings), 3060 (aromatic $\mathrm{C}-\mathrm{H}$ stretching); ${ }^{1} \mathrm{H} \mathrm{NMR}$ $\left(400 \mathrm{MHz}, \mathrm{CDCl}_{3}, \delta\right): 4.04\left(3 \mathrm{H}, \mathrm{s}, \mathrm{OCH}_{3}\right), 7.15-7.88(16 \mathrm{H}, \mathrm{m}, \mathrm{Ar}-\mathrm{H}), 8.37\left(1 \mathrm{H}, \mathrm{s}, \mathrm{C}_{5}{ }^{\prime \prime \prime}-\mathrm{H}\right)$, $8.63\left(1 \mathrm{H}\right.$, poorly resolved d, $\left.\mathrm{C}_{5}{ }^{\prime}-\mathrm{H}\right), 8.71\left(2 \mathrm{H}\right.$, poorly resolved dd, $\mathrm{C}_{2}{ }^{\prime \prime}-\mathrm{H}$ and $\left.\mathrm{C}_{6}{ }^{\prime \prime}-\mathrm{H}\right), 8.98$ $\left(1 \mathrm{H}, \mathrm{s}, \mathrm{C}_{4}-\mathrm{H}\right) ;{ }^{13} \mathrm{C} \mathrm{NMR}\left(100 \mathrm{MHz}, \mathrm{CDCl}_{3}, \delta\right): 56.40\left(\mathrm{OCH}_{3}\right), 116.43\left(\mathrm{C}_{5}{ }^{\prime}\right), 119.32\left(\mathrm{C}_{\mathrm{c}}{ }^{\prime}, \mathrm{C}_{\mathrm{e}}{ }^{\prime}\right)$, 119.52 $\left(\mathrm{C}_{10}\right), 120.17\left(\mathrm{C}_{4}{ }^{\prime \prime \prime}\right), 121.73\left(\mathrm{C}_{8}\right), 123.53\left(\mathrm{C}_{\mathrm{b}}, \mathrm{C}_{\mathrm{f}}\right), 124.65\left(\mathrm{C}_{\mathrm{d}}\right), 125.07\left(\mathrm{C}_{3}\right), 127.06\left(\mathrm{C}_{6}\right)$, 127.47 $\left(\mathrm{C}_{3}{ }^{\prime}\right), 128.66\left(\mathrm{C}_{5}{ }^{\prime \prime}\right), 128.57\left(\mathrm{C}_{5}{ }^{\prime \prime}\right), 129.04\left(\mathrm{C}_{5}\right), 129.56\left(\mathrm{C}_{\mathrm{a}}{ }^{\prime}\right), 132.33\left(\mathrm{C}_{\mathrm{c}}, \mathrm{C}_{\mathrm{e}}\right), 132.87\left(\mathrm{C}_{\mathrm{a}}\right)$, 134.21 $\left(\mathrm{C}_{\mathrm{b}}{ }^{\prime}, \mathrm{C}_{\mathrm{f}}{ }^{\prime}\right), 134.64\left(\mathrm{C}_{4}{ }^{\prime \prime}\right), 139.42\left(\mathrm{C}_{3}{ }^{\prime \prime \prime}\right), 142.30\left(\mathrm{C}_{9}\right), 142.76\left(\mathrm{C}_{7}\right), 148.41\left(\mathrm{C}_{4}\right), 149.45\left(\mathrm{C}_{6}{ }^{\prime \prime}\right)$, 151.12 $\left(\mathrm{C}_{2}{ }^{\prime}\right), 151.34\left(\mathrm{C}_{\mathrm{d}}{ }^{\prime}\right), 154.04\left(\mathrm{C}_{4}{ }^{\prime}\right)$, 154.15( $\left.\mathrm{C}_{6}{ }^{\prime}\right)$, 160.22( $\left.\mathrm{C}_{2}\right)$.; Anal. Calcd. for $\mathrm{C}_{35} \mathrm{H}_{24} \mathrm{~N}_{4} \mathrm{O}_{3}$ C, 76.63; H, 4.41; N, 10.21\%. Found: C, 76.58; H, 4.35; N, 10.28\%.

\section{8-Methoxy-3-[4-(1,3-phenyl-1H-pyrazol-4-yl)-2,4'-bipyridin-6-yl]coumarin (9d):}

Yield: $65 \%$; m.p. $232^{\circ} \mathrm{C}$; IR $\left(\mathrm{KBr}, v_{\max }, \mathrm{cm}^{-1}\right): 1720(\mathrm{C}=\mathrm{O} \delta$-lactone stretching), 1597 and 1458 (aromatic $\mathrm{C}=\mathrm{C}$ and $\mathrm{C}=\mathrm{N}$ stretchings), 3045 (aromatic $\mathrm{C}-\mathrm{H}$ stretching); ${ }^{1} \mathrm{H} \mathrm{NMR}$ $\left(400 \mathrm{MHz}, \mathrm{CDCl}_{3}, \delta\right): 4.03\left(3 \mathrm{H}, \mathrm{s}, \mathrm{OCH}_{3}\right), 7.15-7.88(16 \mathrm{H}, \mathrm{m}, \mathrm{Ar}-\mathrm{H}), 8.38\left(1 \mathrm{H}, \mathrm{s}, \mathrm{C}_{5}{ }^{\prime \prime \prime}-\mathrm{H}\right)$, $8.65\left(1 \mathrm{H}\right.$, poorly resolved $\left.\mathrm{d}, \mathrm{C}_{5}{ }^{\prime}-\mathrm{H}\right), 8.71\left(2 \mathrm{H}\right.$, poorly resolved $\mathrm{dd}, \mathrm{C}_{2}{ }^{\prime \prime}-\mathrm{H}$ and $\left.\mathrm{C}_{6}{ }^{\prime \prime}-\mathrm{H}\right), 8.96$ $\left(1 \mathrm{H}, \mathrm{s}, \mathrm{C}_{4}-\mathrm{H}\right) ;{ }^{13} \mathrm{C} \mathrm{NMR}\left(100 \mathrm{MHz}, \mathrm{CDCl}_{3}, \delta\right): 56.32\left(\mathrm{OCH}_{3}\right), 114.13\left(\mathrm{C}_{5}{ }^{\prime}\right), 119.31\left(\mathrm{C}_{7}\right)$, $119.63\left(\mathrm{C}_{\mathrm{b}}, \mathrm{C}_{\mathrm{f}}\right), 120.01\left(\mathrm{C}_{10}\right), 120.12\left(\mathrm{C}_{4}{ }^{\prime \prime \prime}\right), 120.39\left(\mathrm{C}_{6}\right), 121.07\left(\mathrm{C}_{5}\right), 122.67\left(\mathrm{C}_{\mathrm{d}}\right), 124.53\left(\mathrm{C}_{3}{ }^{\prime}\right)$, 125.14( $\left.\mathrm{C}_{3}\right), 127.06\left(\mathrm{C}_{5}{ }^{\prime \prime}\right), \quad 127.46\left(\mathrm{C}_{5}{ }^{\prime \prime \prime}\right), 128.65\left(\mathrm{C}_{\mathrm{d}}\right), 128.71\left(\mathrm{C}_{\mathrm{b}^{\prime}}, \mathrm{C}_{\mathrm{f}}{ }^{\prime}\right), 128.87\left(\mathrm{C}_{\mathrm{c}^{\prime}}, \mathrm{C}_{\mathrm{e}}{ }^{\prime}\right)$, 129.57( $\left(\mathrm{C}_{\mathrm{c}}, \mathrm{C}_{\mathrm{e}}\right), 132.68\left(\mathrm{C}_{\mathrm{a}}{ }^{\prime}\right), 139.59\left(\mathrm{C}_{\mathrm{a}}\right), 142.65\left(\mathrm{C}_{4}{ }^{\prime \prime}\right), 143.10\left(\mathrm{C}_{4}\right), 143.72\left(\mathrm{C}_{3}{ }^{\prime \prime \prime}\right), 146.44\left(\mathrm{C}_{9}\right)$, 147.00 $\left(\mathrm{C}_{8}\right), 150.14\left(\mathrm{C}_{6}{ }^{\prime \prime}\right), 151.29\left(\mathrm{C}_{2}{ }^{\prime}\right), 151.89\left(\mathrm{C}_{4}{ }^{\prime}\right), 153.82\left(\mathrm{C}_{6}{ }^{\prime}\right), 159.59\left(\mathrm{C}_{2}\right)$; Anal. Calcd. for $\mathrm{C}_{35} \mathrm{H}_{24} \mathrm{~N}_{4} \mathrm{O}_{3} \mathrm{C}, 76.63 ; \mathrm{H}, 4.41 ; \mathrm{N}, 10.21 \%$. Found: $\mathrm{C}, 76.55 ; \mathrm{H}, 4.50 ; \mathrm{N}, 10.27 \%$.

8-Methoxy-3-[4-(1-phenyl-3-p-tolyl-1H-pyrazol-4-yl)-2,4'-bipyridin-6-yl]coumarin (9e): Yield: $78 \%$; m.p. $184^{\circ} \mathrm{C}$; IR $\left(\mathrm{KBr}, v_{\max }, \mathrm{cm}^{-1}\right)$ : $1716(\mathrm{C}=\mathrm{O} \delta$-lactone stretching $), 1592$ and 1476 (aromatic $\mathrm{C}=\mathrm{C}$ and $\mathrm{C}=\mathrm{N}$ stretchings), 3023 (aromatic $\mathrm{C}-\mathrm{H}$ stretching); ${ }^{1} \mathrm{H}$ NMR 
(400MHz, $\left.\mathrm{CDCl}_{3, \delta}\right): 2.44\left(3 \mathrm{H}, \mathrm{s}, \mathrm{CH}_{3}\right), 4.02\left(3 \mathrm{H}, \mathrm{s}, \mathrm{OCH}_{3}\right), 7.14-7.86(15 \mathrm{H}, \mathrm{m}, \mathrm{Ar}-\mathrm{H}), 8.34$ $\left(1 \mathrm{H}, \mathrm{s}, \mathrm{C}_{5}{ }^{\prime \prime \prime}-\mathrm{H}\right), 8.63\left(1 \mathrm{H}\right.$, poorly resolved $\left.\mathrm{d}, \mathrm{C}_{5}{ }^{\prime}-\mathrm{H}\right), 8.70\left(2 \mathrm{H}\right.$, poorly resolved dd, $\mathrm{C}_{2}{ }^{\prime \prime}-\mathrm{H}$ and $\left.\mathrm{C}_{6}{ }^{\prime \prime}-\mathrm{H}\right), 8.93\left(1 \mathrm{H}, \mathrm{s}, \mathrm{C}_{4}-\mathrm{H}\right) ;{ }^{13} \mathrm{C} \mathrm{NMR}\left(100 \mathrm{MHz}, \mathrm{CDCl}_{3}, \delta\right): 21.37\left(\mathrm{CH}_{3}\right), 56.30\left(\mathrm{OCH}_{3}\right)$, $114.08\left(\mathrm{C}_{5}{ }^{\prime}\right), 119.25\left(\mathrm{C}_{7}\right), 119.62\left(\mathrm{C}_{\mathrm{b}}, \mathrm{C}_{\mathrm{f}}\right), 119.92\left(\mathrm{C}_{10}\right), 120.13\left(\mathrm{C}_{4}{ }^{\prime \prime \prime}\right), 120.38\left(\mathrm{C}_{6}\right), 121.03\left(\mathrm{C}_{5}\right)$, $122.64\left(\mathrm{C}_{\mathrm{d}}\right), \quad 124.51\left(\mathrm{C}_{3}{ }^{\prime}\right), \quad 125.18\left(\mathrm{C}_{3}\right), \quad 126.95\left(\mathrm{C}_{5}{ }^{\prime \prime}\right), \quad 127.39\left(\mathrm{C}_{5}{ }^{\prime \prime \prime}\right), \quad 128.70\left(\mathrm{C}_{\mathrm{c}}{ }^{\prime}, \mathrm{C}_{\mathrm{e}}{ }^{\prime}\right)$, 129.37( $\left(\mathrm{C}_{\mathrm{b}}{ }^{\prime}, \quad \mathrm{C}_{\mathrm{f}}{ }^{\prime}\right), \quad 129.53\left(\mathrm{C}_{\mathrm{c}}, \mathrm{C}_{\mathrm{e}}\right), \quad 129.75\left(\mathrm{C}_{\mathrm{a}}{ }^{\prime}\right), \quad 138.45\left(\mathrm{C}_{\mathrm{d}}{ }^{\prime}\right), \quad 139.62\left(\mathrm{C}_{\mathrm{a}}\right), \quad 142.78\left(\mathrm{C}_{4}{ }^{\prime \prime}\right)$, 143.04 $\left(\mathrm{C}_{4}\right), 143.69\left(\mathrm{C}_{3}{ }^{\prime \prime \prime}\right), 146.26\left(\mathrm{C}_{9}\right), 146.98\left(\mathrm{C}_{8}\right), 150.35\left(\mathrm{C}_{6}{ }^{\prime \prime}\right), 151.28\left(\mathrm{C}_{2}{ }^{\prime}\right), 151.79\left(\mathrm{C}_{4}{ }^{\prime}\right)$, 153.84 $\left(\mathrm{C}_{6}{ }^{\prime}\right), 159.70\left(\mathrm{C}_{2}\right.$ of coumarin); Anal. Calcd. for $\mathrm{C}_{36} \mathrm{H}_{26} \mathrm{~N}_{4} \mathrm{O}_{3} \mathrm{C}, 76.85 ; \mathrm{H}, 4.66 ; \mathrm{N}$, 9.96\%. Found: C, 76.78; H, 4.72; N, 9.89\%.

\section{8-Methoxy-3-\{4-[3-(4-methoxyphenyl)-1-phenyl-1H-pyrazol-4-yl]-2,4'-bipyridin-6-yl\} coumarin (9f):}

Yield: $68 \%$; m.p. $244^{\circ} \mathrm{C}$; IR $\left(\mathrm{KBr}, v_{\max }, \mathrm{cm}^{-1}\right)$ : $1720(\mathrm{C}=\mathrm{O} \delta$-lactone stretching), 1590 and 1460 (aromatic $\mathrm{C}=\mathrm{C}$ and $\mathrm{C}=\mathrm{N}$ stretchings), 3058 (aromatic $\mathrm{C}-\mathrm{H}$ stretching); ${ }^{1} \mathrm{H}$ NMR $\left(400 \mathrm{MHz}, \mathrm{CDCl}_{3}, \delta\right): 3.88\left(3 \mathrm{H}, \mathrm{s}, \mathrm{OCH}_{3}\right), 4.04\left(3 \mathrm{H}, \mathrm{s}, \mathrm{OCH}_{3}\right), 6.97-8.35(15 \mathrm{H}, \mathrm{m}, \mathrm{Ar}-\mathrm{H}), 8.34$ $\left(1 \mathrm{H}, \mathrm{s}, \mathrm{C}_{5}{ }^{\prime \prime \prime}-\mathrm{H}\right), 8.65\left(1 \mathrm{H}\right.$, poorly resolved d, $\left.\mathrm{C}_{5}{ }^{\prime}-\mathrm{H}\right), 8.71\left(2 \mathrm{H}\right.$, poorly resolved dd, $\mathrm{C}_{2}{ }^{\prime \prime}-\mathrm{H}$ and $\left.\mathrm{C}_{6}{ }^{\prime \prime}-\mathrm{H}\right), 8.95\left(1 \mathrm{H}, \mathrm{s}, \mathrm{C}_{4}-\mathrm{H}\right) ;{ }^{13} \mathrm{C} \mathrm{NMR}\left(100 \mathrm{MHz}, \mathrm{CDCl}_{3}, \delta\right): 55.40\left(\mathrm{OCH}_{3}\right), 56.33\left(\mathrm{OCH}_{3}\right)$, 114.17( $\left(\mathrm{C}_{5}{ }^{\prime}\right), \quad 119.25\left(\mathrm{C}_{7}\right), \quad 119.66\left(\mathrm{C}_{\mathrm{c}^{\prime}}, \mathrm{C}_{\mathrm{e}}{ }^{\prime}\right), \quad 119.76\left(\mathrm{C}_{\mathrm{b}}, \mathrm{C}_{\mathrm{f}}\right), \quad 120.14\left(\mathrm{C}_{4}{ }^{\prime \prime \prime}\right), \quad 120.39\left(\mathrm{C}_{6}\right)$, 121.16 $\left(\mathrm{C}_{5}\right), 122.81\left(\mathrm{C}_{\mathrm{d}}\right), 124.54\left(\mathrm{C}_{3}{ }^{\prime}\right), 125.05\left(\mathrm{C}_{3}\right), 125.21\left(\mathrm{C}_{\mathrm{a}}{ }^{\prime}\right), 126.95\left(\mathrm{C}_{5}{ }^{\prime \prime}\right), 127.42\left(\mathrm{C}_{5}{ }^{\prime \prime \prime}\right)$, $129.55\left(\mathrm{C}_{\mathrm{c}}, \mathrm{C}_{\mathrm{e}}\right), \quad 130.07\left(\mathrm{C}_{\mathrm{b}}{ }^{\prime}, \mathrm{C}_{\mathrm{f}}{ }^{\prime}\right), \quad 139.64\left(\mathrm{C}_{\mathrm{a}}\right), 142.94\left(\mathrm{C}_{4}{ }^{\prime \prime}\right), \quad 143.10\left(\mathrm{C}_{4}\right), \quad 143.74\left(\mathrm{C}_{3}{ }^{\prime \prime \prime}\right)$, 146.65 $\left(\mathrm{C}_{9}\right), 147.01\left(\mathrm{C}_{8}\right), 149.98\left(\mathrm{C}_{6}{ }^{\prime \prime}\right), 151.01\left(\mathrm{C}_{2}{ }^{\prime}\right), 151.94\left(\mathrm{C}_{\mathrm{d}}{ }^{\prime}\right), 153.77\left(\mathrm{C}_{4}{ }^{\prime}\right), 159.68\left(\mathrm{C}_{6}{ }^{\prime}\right)$, 160.01( $\left.\mathrm{C}_{2}\right)$; Anal. Calcd. for $\mathrm{C}_{36} \mathrm{H}_{26} \mathrm{~N}_{4} \mathrm{O}_{4} \mathrm{C}, 74.73 ; \mathrm{H}, 4.53 ; \mathrm{N}, 9.68 \%$. Found: C, 74.79; $\mathrm{H}$, $4.60 ; \mathrm{N}, 9.74 \%$.

\section{2. Biological assay}

All the newly synthesized target compounds were evaluated for their in vitro antibacterial activity against Staphylococcus aureus (MTCC 96) and Bacillus subtilis (MTCC 441) as examples of Gram positive bacteria and Escherichia coli (MTCC 443) and Salmonella typhi (MTCC 98) as examples of Gram negative bacteria. They were also evaluated for their in vitro antifungal activity against Candida albicans (MTCC 227) and Aspergillus niger (MTCC 282) as fungal strains. For comparison, the standard drug used for antibacterial potency of the compounds was ampicillin, a broad spectrum antibiotic, while the drugs used for antifungal potency of the compounds were griseofulvin and nystatin. The screening results (Table 1) indicated that all the tested compounds exhibited different inhibitory effects against different test organisms. All MTCC cultures were collected from Institute of Microbial Technology, Chandigarh and tested against above mentioned known drugs. Mueller Hinton broth was used as nutrient medium to grow and dilute the drug suspension for the test. Inoculum's size for test strain was adjusted to $10^{8} \mathrm{CFU}$ (Colony Forming Unit) per milliliter by comparing the turbidity. The newly prepared compounds were screened for their MICs by broth microdilution method. DMSO was used as a diluent to get desired concentration of compounds to test upon standard bacterial strains. Serial dilutions were prepared in primary and secondary screening. The control tube containing no antibiotic was immediately subcultured (before inoculation) by spreading a loopful evenly over a quarter of plate of medium suitable for the growth of the test organism and put for incubation at $37^{\circ} \mathrm{C}$ overnight. The tubes were then incubated overnight. The MIC of the control organism was read to check the accuracy of the compound concentrations The lowest concentration (highest dilution) required to arrest the growth of bacteria was regarded as minimum inhibitory concentration (MIC). All the tubes showing no visible growth (same as control 
tube) were sub cultured and incubated overnight at $37^{\circ} \mathrm{C}$. The amount of growth from the control tube before incubation (which represents the original inoculum) was compared. Subcultures might show (i) similar number of colonies indicating bacteriostatic (ii) a reduced number of colonies indicating a partial or slow bactericidal activity (iii) no growth if the whole inoculum has been killed. The test must include a second set of the same dilutions inoculated with an organism of known sensitivity. Each synthesized compound was diluted obtaining $2000 \mu \mathrm{g} / \mathrm{mL}$ concentration as a stock solution. In primary screening 500, 250 and $200 \mu \mathrm{g} / \mathrm{mL}$ concentrations of the synthesized compounds were taken. The compounds which found active in this primary screening were further tested in a second set of dilution using $100,62.5,50$ and $25 \mu \mathrm{g} / \mathrm{mL}$ concentrations against all microorganisms. The highest dilution showing at least $99 \%$ inhibition is taken as MIC.

\section{CONCLUSION}

Present study described successful hybridization strategy of three bioactive moieties, pyridyl substituted coumarin, bipyridine and pyrazolyl substituted pyridine in single scaffold. The target compounds were synthesized in good yield by adopting Krohnke's protocol. Majority of the compounds were found to be active against Staphylococcus aureus and Bacillus subtilis. In antifungal activity, majority of the compounds showed excellent activity against Candida albican as compared to griseofulvin. Antimicrobial screening results revealed that compounds $\mathbf{3 b}, \mathbf{3 e}, \mathbf{7 b}, \mathbf{8 b}, \mathbf{8 c}$ and $9 \mathbf{b}$ were found to be the most proficient members of the series. Reviewing the antimicrobial data, it is worth mentioning here that coumarins bearing bipyridine and pyrazole entities as substitution serve as promising lead scaffolds for further generation of new antimicrobial agents.

\section{ACKNOWLEDGEMENT}

The authors are thankful to UGC for financial assistance under RFSMS. The authors are also thankful to the Department of Chemistry, Sardar Patel University for providing research facilities.

\section{References}

[1] Neu, H.C. The Crisis in Antiboitic Resistance. Science, 1992, 257(5073), 1064-1072.

[2] Opar, A. Bad Bugs Needs more Drugs. Nat. Rev. Drug Discovery, 2007, 6(12), 943-944.

[3] Anderson, A.C; Schiffer, C; Pollastri, M and Peet, N.P. The challenge of developing robust drugs to overcome resistance. Drug Discovery Today, 2011, 16(17-18), 755-761.

[4] Todd, B. Beyond MRSA: VISA and VRSA: what will ward off these pathogens in health care facilities? Am. J. Nurs., 2006, 106(4), 28-30.

[5] Moffett, R.B. Central nervous system depressants VII. Pyridyl coumarins. J. Med. Chem., 1964, 7, 446-449.

[6] Garazd, Y.L; Kornienko, E.M; Maloshtan; L.N; Garazd, M.M; Khilya, V.P. Modified coumarins. 17. Synthesis and anticoagulant activity of 3,4-cycloannelated coumarin Dglycopyranosides. Chem. Nat. Prod., 2005, 41, 508-512. 
[7] Sreenivasulu, B; Sundaramurthy, V; Subba Rao, N.V. Search for physiologically active compounds. Part XXIII. Synthesis of 3-(3-pyridyl) and 3-(3-pyridyl)-4-methyl coumarins. Proc. Indian. Acad. Sci. A, 1974, 79, 41-48.

[8] Moffett, R. B. Pyridylcoumarins. U. S. Patent, 3, 201, 406, 1965.

[9] Matos, M.J; Vilar, Gonzalez-Franco, R.M; Uriarte, E; Santana, L; Friedman, C; Tatonetti, N.P; Viña, D and Fontenla, J.A. Novel (coumarin-3-yl)carbamates as selective MAO-B inhibitors: Synthesis, in vitro and in vivo assays, theoretical evaluation of ADME properties and docking study. Eur. J. Med. Chem., 2013, 63, 151-161.

[10] Lad, H.B; Giri, R.R and Brahmbhatt, D.I. An efficient synthesis of some new 3bipyridinyl substituted coumarins as potent antimicrobial agents. Chin. Chem. Lett., 2013, 24, 227-229.

[11] Bhila, V.G; Patel, C.V; Brahmbhatt, D.I. One pot synthesis of some novel coumarins containing 5-(substituted-2-hydroxybenzoyl) pyridine as a new class of antimicrobial and antituberculosis agents. Med. Chem .Res., 2013, 22(9), 4338-4346.

[12] Shimizu, T; Nozawa, T; Hatano, M. Magnetic circular dichroism studies of pyridineheme complexes in aqueous media. Bioinorg. Chem., 1976, 6(2), 119-131.

[13] Jia, J; Hubberstey, P; Champness, N.R and Schröder, M. In: Molecular Networks (Struct Bond); Mir Wais Hosseini, Ed.; Springer: Verlag Berlin Heidelberg, 2009; Vol. 132, pp. 135-161.

[14] Fraser, C.L and Smith, A.P. Metal complexes with polymeric ligands: Chelation and metalloinitiation approaches to metal tris(bipyridine) containing materials. J. Polym. Sci., Part A: Polym. Chem., 2000, 38(S1), 4704-4716.

[15] Mongin, F; Trécourt, F; Gervais, B; Mongin, O; Quéguiner, G. First Synthesis of Caerulomycin B. A New Synthesis of Caerulomycin C. J. Org. Chem., 2002, 67, 3272-3276.

[16] El-Kemary, M; Organero, J.A; Douhal, A. Fast Relaxation Dynamics of the Cardiotonic Drug Milrinone in Water Solutions. J. Med. Chem., 2006, 49(11), 3086-3091.

[17] Patel, M.N; Dosi, P.A; Bhatt, B.S. Square planar palladium (II) complexes of bipyridines: synthesis, characterization and biological studies. J. Coord. Chem., 2012, 65(21), 3833-3844.

[18] Patel, M.N; Parmar, P.A; Gandhi, D.S. DNA interactions and cytotoxic studies of cisplatin analogues of substituted 2,2-bipyridines, Spectrochim. Acta., 2012, 97C, 54-59.

[19] Dai, H; Yu, H; Liu, J; Li, Y; Qin, X; Zhang, X; Qin, Z; Wang, T; Fang, J. Synthesis and bioactivities of novel trifluoromethylated pyrazole oxime ether derivatives containing a pyridyl moiety. Arkivoc., 2009(vii), 126-142.

[20] Johns, B.A; Gudmundsson, K.A; Allen, S.H. Pyrazolo[1,5-a]pyridine antiherpetics: Effects of the $\mathrm{C} 3$ substituent on antiviral activity. Bioorg. Med. Chem. Lett., 2007, $17(10), 2858-2862$. 
[21] Robertson, D.W; Krushinski, J.H; Pollock, G.D; Haye, J.S. Imidazole-pyridine bioisosterism: comparison of the inotropic activities of pyridine- and imidazolesubstituted 6-phenyldihydropyridazinone cardiotonics. J. Med. Chem., 1988, 31, 461-465.

[22] Thomas, K.D; Adhikari, A.V; Shetty, N.S. Design, synthesis and antimicrobial activities of some new quinoline derivatives carrying 1,2,3-triazole moiety. Eur. J. Med. Chem., 2010, 45(9), 3803-3810.

[23] Krohnke, F. The specific synthesis of pyridines and oligopyridines, Synthesis, 1976, 1, 1-24.

[24] NCCLS (National Committee for Clinical Laboratory Standards), Performance Standards for Antimicrobial Susceptibility Testing: Twelfth Informational Supplement. 2002, 1-56238-454-6, M100-S12 (M7).

[25] Biscler, A. Ueber die entstehung einiger substituirter indole. Chem Ber., 1892, 25, 2860-2879.

[26] Kira, M.A; Abdel-Raeman, M.O; Gadalla, K.Z. The vilsmeier-haack reaction - III Cyclization of hydrazones to pyrazoles. Tetrahedron. Lett., 1969, 10(2), 109-110.

[27] Basnet, A.; Thapa, P.; Karki, R.; Na, Y.; Jhang, Y.; Jeong, B. S.; Jeong, T. C.; Lee, C. S.; Lee, E. S. 2,4,6-Trisubstituted pyridines: synthesis, topoisomerase I and II inhibitory activity, cytotoxicity, and structure-activity relationship. Bioorg. Med. Chem., 2007, 15(13), 4351-4359. 